\title{
Stochastic phase resetting of two coupled phase oscillators stimulated at different times
}

\author{
Peter A. Tass \\ Institute of Medicine, Research Center Jülich, 52425 Jülich, Germany \\ and Department of Stereotaxic and Functional Neurosurgery, University Hospital, 50924 Cologne, Germany
}

(Received 21 January 2003; published 6 May 2003)

\begin{abstract}
A model of two coupled phase oscillators is presented, where the oscillators are subject to random forces and are stimulated at different times. Transient phase dynamics, synchronization, and desynchronization, which are stimulus locked (i.e., tightly time locked to a repetitively administered stimulus), are investigated. Complex coordinated responses, in terms of a noise-induced switching across trials between qualitatively different responses, may occur when the two oscillators are reset close to an unstable fixed point of their relative phases. This can be achieved with an appropriately chosen delay between the two stimuli. The switching of the responses shows up as a coordinated cross-trial (CT) response clustering of the oscillators, where the two oscillators produce two different pairs of responses. By varying noise amplitude and coupling strength we observe a stochastic resonance and a coupling-mediated resonance of the CT response clustering, respectively. The presented data analysis method makes it possible to detect such processes in numerical and experimental signals. Its time resolution is enormous, since it is only restricted by the time resolution of the preprocessing necessary for extracting the phases from experimental data. In contrast, standard data analysis tools applied across trials relative to stimulus onset, such as CT averaging (where an ensemble of poststimulus responses is simply averaged), CT standard deviation, and CT cross correlation, fail in detecting complex coordinated responses and lead to severe misinterpretations and artifacts. The consequences for the analysis of evoked responses in medicine and neuroscience are significant and are discussed in detail.
\end{abstract}

DOI: 10.1103/PhysRevE.67.051902

\section{INTRODUCTION}

Synchronization abounds in physics [1], chemistry [2], biology [3], neuroscience [4], and medicine [5,6]. In the past years, numerous studies addressed the stochastic phase synchronization of periodic oscillators $[2,1]$ and chaotic oscillators [7] in physics $[1,8]$, chemistry [9], and biology $[10,11]$. Various dynamical properties of stationary synchronization processes have been revealed, such as stochastic resonance of phase synchronization [12]. The majority of these studies were dedicated to stationary synchronization processes evolving on a long time scale, for time $t \rightarrow \infty$. In this context stochastic phase synchronization was defined as the appearance of one or more prominent peaks in the distribution of the phase difference during a sufficiently long observation $[7,13]$. Put otherwise, the hallmark of phase synchronization is the tendency of the oscillators to maintain a stable phase relationship.

On the other hand, in physics and biology there is a large number of important dynamical processes that are caused by pulsatile stimuli. Such processes are transient and act on short time scales. In particular, they violate a $t \rightarrow \infty$ as well as a quasistationarity assumption. To cope with stimulus-locked transient dynamics of this kind, we use an approach based on stochastic phase resetting $[5,16,17]$ : Stimulus-locked phase dynamics or stimulus-locked synchronization means that a particular transient dynamics of the phases or of the $n: m$ phase difference is stimulus locked, i.e., tightly locked in time to a repetitively delivered stimulus. More precisely, stochastic stimulus-locking of the phase dynamics and stochastic stimulus-locked $n: m$ phase synchronization are characterized by the presence of one or more prominent peaks in the distribution of the phases and of the $n: m$ phase difference across trials at each time $t$ relative to stimulus onset in an
PACS number(s): 87.19.La, 05.45.Xt, 05.40.Ca

ensemble of responses. The relevant criterion here is a stereotypical time course of the phases or the $n: m$ phase difference relative to stimulus onset across trials.

We apply this approach to study transient stimulus-locked phase dynamics, synchronization, and desynchronization in two coupled phase oscillators, which are subject to random forces and stimulated at different times. In a previous study on two coupled and simultaneously stimulated phase oscillators it has been shown that complex coordinated responses occur, provided the stimuli are appropriately chosen $[16,17]$. For example, an antiphase reset of in-phase synchronized oscillators causes a noise-induced switching between qualitatively different responses relative to stimulus onset. For this, however, the two phase-dependent stimuli have to be of a particular form. If the stimulus of oscillator 1 (added to the right-hand side of the evolution equation of the phase) reads, e.g., $S_{1}\left(\psi_{1}\right)=I \cos \psi_{1}$, the stimulation mechanism of oscillator 2 has to take the complementary form $S_{2}\left(\psi_{2}\right)=\cos \left(\psi_{2}\right.$ $+\pi)$, where the phase shift of $\pi$ in the argument causes the antiphase reset (see below).

If both oscillators model identical units, such as identical oscillatory neurons, it might be difficult to realize such complementary stimuli experimentally. In this paper, we show that the noise-induced switching between qualitatively different responses may emerge in a much more general setting. We may stimulate identical oscillators with identical stimuli and produce such complex responses, provided we stimulate the oscillators at different times with an appropriately chosen delay. From an experimental point of view, varying the delay between two stimuli is trivial compared to modifying the stimulation mechanism.

We shall see that stimulation at different times and stimulation with antiphase stimuli have several features in common. For example, in both cases we observe a stochastic 
resonance that causes the noise-induced switching between different responses across trials. On the other hand, only in the case of stimulation at different times strong enough coupling may prevent the oscillators from such complex responses. In this case, we even observe a pronounced coupling-mediated resonance of the noise-induced switching.

The study of stimulus-induced transient responses of oscillators by itself is an interesting topic. Furthermore, it has several significant applications in various fields of physics and natural sciences. The investigation of transient shortterm brain responses evoked by sensory stimuli is a key approach for the study of cerebral information processing and diagnosis [6]. For this purpose, we may apply three standard data analysis methods across trials.

(i) A stimulus-locked response of a neuronal population is typically analyzed with a cross-trial (CT) averaging, where an ensemble of poststimulus responses is averaged across trials relative to stimulus onset $[14,15]$. The interactions of the oscillators are typically guessed by phenomenologically studying such CT averaged responses, e.g., by comparing changes of peak latencies or amplitudes under varying conditions.

(ii) To determine how stereotypical the responses are, we may calculate a standard deviation across trials relative to stimulus onset.

(iii) To detect linear correlations in an ensemble of pairs of responses, we may use a CT cross correlation, i.e., a cross correlation calculated across all trials at each time $t$ relative to stimulus onset.

Instead of these CT standard analysis techniques, we use a stochastic phase resetting analysis $[5,16,17]$. For this, we determine the time-dependent distributions of the phases and of the $n: m$ phase difference calculated across trials for each time $t$ relative to stimulus onset, and evaluate these distributions statistically. In this way it is possible to detect the noise-induced switching between different responses across trials. In contrast, the CT standard techniques-CT averaging, CT standard deviation, and CT cross correlation-lead to misinterpretations and even artifacts. Our results have severe consequences, since the CT standard methods are used for the analysis of evoked responses in neuroscience and medicine. The gold standard for the extraction of stimuluslocked responses, the CT averaging, is even a major tool for diagnosis [6]. We shall discuss in detail how we can avoid artifacts originating from CT averaging by applying the proposed data analysis techniques.

\section{STOCHASTIC MODEL}

We consider a model given by two phase oscillators with phases $\psi_{1}, \psi_{2}$ and constant amplitudes governed by

$$
\dot{\psi}_{j}=\omega_{j}-K \sin \left(\psi_{j}-\psi_{k}\right)+X_{j}(t) S_{j}\left(\psi_{j}\right)+F_{j}(t),
$$

where $j, k=1,2$ and $j \neq k$. The eigenfrequencies read $\omega_{1,2}$ $=\omega \pm \gamma / 2$ with detuning $\gamma$. The stimuli are modeled by $2 \pi$-periodic, time-independent functions $S_{j}\left(\psi_{j}\right)=S_{j}\left(\psi_{j}\right.$ $+2 \pi$ ). In several fields of the natural sciences, and typically in biology, the effect of a stimulus is phase dependent [3]. A
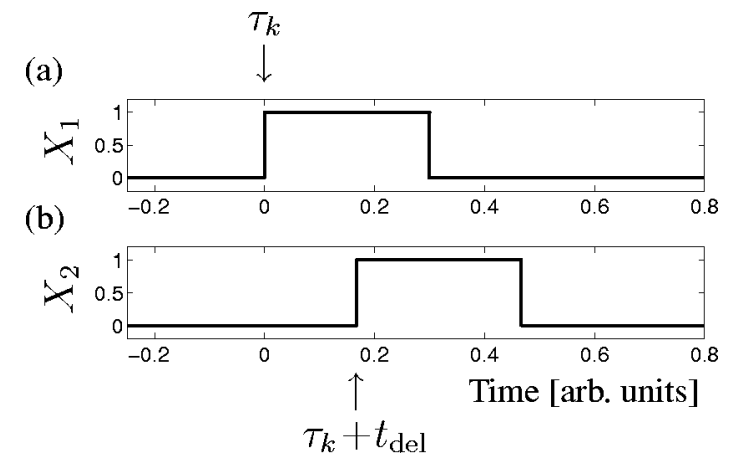

FIG. 1. Time course of $X_{j}$ from Eq. (2) during stimulation. Oscillator 1 is stimulated first at time $\tau_{k}=0$ (a), while the onset of the stimulus of oscillator 2 is delayed by $t_{\mathrm{del}}(\mathrm{b})$. Both stimuli are of equal duration. Therefore, $t_{\text {del }}$ is also the delay between the offsets of the two stimuli. This pair of stimuli is administered at random times $\tau_{1}, \tau_{2}, \ldots, \tau_{l}$.

pair of stimuli is administered as illustrated in Fig. 1: Oscillator 1 is stimulated first, while the onset of the stimulus of oscillator 2 is delayed by $t_{\mathrm{del}} \geqslant 0$. We assume that the duration of both stimuli is the same. Accordingly, $t_{\mathrm{del}}$ is also the delay between the offsets of the two stimuli. An extension to stimuli of different duration is straightforward and is discussed in Sec. X. Switching on and off the stimulus of oscillator $j$ is modeled by

$$
X_{j}(t)= \begin{cases}1: & \text { stimulus is on at time } t \\ 0: & \text { stimulus is off at time } t,\end{cases}
$$

where $j=1,2$ and $X_{1}(t)=X_{2}\left(t+t_{\text {del }}\right)$. The random forces $F_{1}$ and $F_{2}$ are the Gaussian white noise fulfilling $\left\langle F_{j}(t)\right\rangle=0$ and $\left\langle F_{j}(t) F_{k}(\tilde{t})\right\rangle=D \delta_{j k} \delta(t-\widetilde{t})$ with constant noise amplitude $D$. Equation (1) may serve as a minimal model for two electrically stimulated neurons [5] or as a minimal model for two neuronal populations affected by sensory stimuli as explained below. We set the amplitude of both oscillators equal to 1 and define the signal of the $j$ th phase oscillator as

$$
x_{j}(t)=\cos \psi_{j}(t) \text {. }
$$

\section{CROSS-TRIAL ANALYSIS BASED ON STOCHASTIC PHASE RESETTING}

We introduce normalized phases

$$
\phi_{j}(t)=\frac{\psi_{j}(t)}{2 \pi} \bmod 1(j=1,2)
$$

and the normalized cyclic $n: m$ phase difference

$$
\varphi_{n, m}(t)=\frac{n \psi_{1}(t)-m \psi_{2}(t)}{2 \pi} \bmod 1 .
$$

The goal of our analysis is to detect whether in an ensemble of responses to the stimulus there are epochs during which phases $\phi_{1}, \phi_{2}$ and/or the phase difference $\varphi_{n, m}$ display a stereotypical, tightly stimulus-locked time course. For this, at random times $\tau_{1}, \tau_{2}, \ldots, \tau_{l}$ we deliver a series of $l$ identical pairs of stimuli shown in Fig. 1. In particular, in each pair of 
stimuli the time delay $t_{\text {del }}$ between the onsets of the first and second stimulus is kept constant throughout the whole series. The length of the interstimulus intervals is randomized according to

$$
\tau_{k+1}-\tau_{k}=t_{\mathrm{win}}+\zeta_{k}
$$

where $t_{\text {win }}$ is constant and large compared to the stimulation duration as well as the time scale of the transient dynamics. $\zeta_{k}$ is uniformly distributed in $[0,2 \pi / \omega]$. To each stimulus we attach an identical time window $\left[t_{a}, t_{b}\right]\left(t_{a}<0, t_{b}>0\right.$, Fig. $2)$. Each window has a time axis $t^{\prime}$, so that $t^{\prime} \in\left[t_{a}, t_{b}\right]$, where the onset of the stimulus in each window lies in $t^{\prime}$ $=0$. The window length $t_{b}-t_{a}$ is smaller than the length of the interstimulus intervals $\left(t_{b}-t_{a}<t_{\text {win }}\right)$, but is large compared to the time scale of the transient dynamics.

For the sake of simplicity let us drop the prime in $t^{\prime}$, and keep in mind that from now on, $t$ denotes the time axis of the window. To study the dynamics of the ensemble of stimuluslocked responses for each time $t \in\left[t_{a}, t_{b}\right]$, we introduce the time-dependent cross-trial $(C T)$ distributions of the normalized phases from Eq. (4) and the cyclic $n: m$ phase difference from Eq. (5) by

$$
\left\{\phi_{j}\left(t+\tau_{k}\right)\right\}_{k=1, \ldots, l}, \quad\left\{\varphi_{n, m}\left(t+\tau_{k}\right)\right\}_{k=1, \ldots, l} .
$$

The time course of $\phi_{j}$ and $\varphi_{n, m}$ is perfectly stimulus locked at time $t$ if the corresponding CT distributions from Eq. (7) are Dirac-like distributions, i.e., $\phi_{j}\left(t+\tau_{i}\right)=\phi_{j}\left(t+\tau_{k}\right)$ and $\varphi_{n, m}\left(t+\tau_{i}\right)=\varphi_{n, m}\left(t+\tau_{k}\right)$ for all $i, k=1, \ldots, l$. On the other hand, if $\phi_{j}$ and $\varphi_{n, m}$ are not at all stimulus locked at time $t$, these distributions are uniform. The extent of stimulus locking of $\phi_{j}$ and $\varphi_{n, m}$ is quantified for each time $t$ by means of the time-dependent stimulus-locking indices $\lambda_{j}^{(\nu)}(t)$ of $\phi_{j}$ given by

$$
\lambda_{j}^{(\nu)}(t)=\left|\frac{1}{l} \sum_{k=1}^{l} \exp \left[i \nu 2 \pi \phi_{j}\left(\tau_{k}+t\right)\right]\right|,
$$

and the n:m stimulus locking indices $\sigma_{n, m}^{(\nu)}(t)$ of $\varphi_{n, m}$ given by

$$
\sigma_{n, m}^{(\nu)}(t)=\left|\frac{1}{l} \sum_{k=1}^{l} \exp \left[i \nu 2 \pi \varphi_{n, m}\left(\tau_{k}+t\right)\right]\right|
$$

where $|y|$ denotes the modulus of $y$, and $\nu$ is an integer $[16,17] . \lambda_{j}^{(\nu)}(t)$ and $\sigma_{n, m}^{(\nu)}(t)$ detect whether $\phi_{j}$ 's or $\varphi_{n, m}$ 's CT distribution from Eq. (7) at time $t$ has $\nu$ peaks that are equally spaced in $[0,1]$ (modulo 1) and fulfill $0 \leqslant \lambda_{j}^{(\nu)}(t)$ $\leqslant 1,0 \leqslant \sigma_{n, m}^{(\nu)}(t) \leqslant 1$ for $t \in\left[t_{a}, t_{b}\right]$ and for all integer $\nu$.

Let us consider the three leading indices $(\nu=1,2,3)$ in four different situations.

(i) If the distribution $\left\{\phi_{j}\left(t+\tau_{k}\right)\right\}_{k=1, \ldots, l}$ at time $t$ is uniform, then $\lambda_{j}^{(\nu)}(t)=0$ for $\nu=1,2,3$.

(ii) One pronounced peak of the distribution $\left\{\phi_{j}(t\right.$ $\left.\left.+\tau_{k}\right)\right\}_{k=1, \ldots, l}$ at time $t$ corresponds to large $\lambda_{j}^{(\nu)}(t)$ for $\nu$ $=1,2,3$. (iii) Two pronounced antiphase peaks of the distribution $\left\{\phi_{j}\left(t+\tau_{k}\right)\right\}_{k=1, \ldots, l}$ at time $t$ are characterized by large $\lambda_{j}^{(2)}(t)$ and small $\lambda_{j}^{(\nu)}(t)$ for $\nu=1,3$. Two symmetric

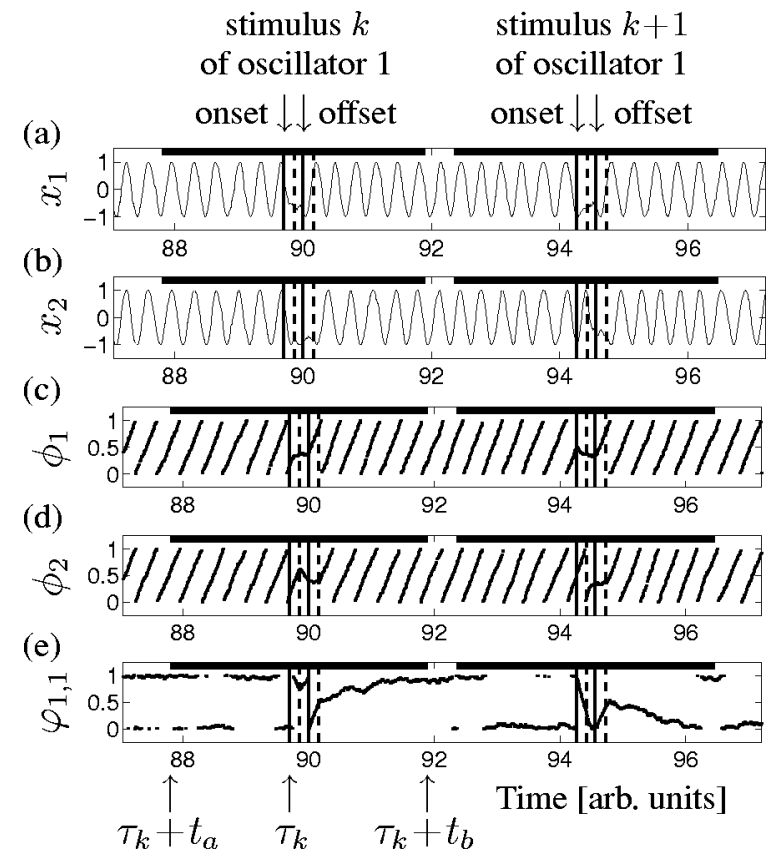

FIG. 2. The scheme illustrates the cross-trial analysis, where a series of $l$ identical pairs of stimuli from Fig. 1 is administered at random times $\tau_{1}, \tau_{2}, \ldots, \tau_{l}$. Each pair consists of a stimulus administered to oscillator 1 (with onset $\tau_{k}$ ) followed by a stimulus administered to oscillator 2 after a constant delay $t_{\text {del }}$ (with onset $\left.\tau_{k}+t_{\mathrm{del}}\right)$, where $t_{\mathrm{del}}$ here equals half a period of the oscillation. Onsets and offsets of the stimuli of oscillator 1 are indicated by solid vertical lines, whereas onsets and offsets of the stimuli of oscillator 2 are denoted by dashed vertical lines, respectively. An identical time window $\left[t_{a}, t_{b}\right]$ (with $t_{a}<0, t_{b}>0$ ) is attached to each stimulus and indicated by a shaded region at the top of each panel. The signals $x_{1}(t)=\cos \left[2 \pi \phi_{1}(t)\right]$ and $x_{2}(t)=\cos \left[2 \pi \phi_{2}(t)\right]$ from Eq. (3) are shown in (a) and (b). The corresponding phases $\phi_{1}$ and $\phi_{2}$ from Eq. (4) are displayed in (c) and (d). The normalized cyclic 1:1 phase difference $\varphi_{1,1}$ from Eq. (5) is shown in (e). Note, $\varphi_{1,1}=0$ and $\varphi_{1,1}=1$ are identical, so that continuous variations around $\varphi_{1,1}=0$ appear as abrupt jumps between 0 and 1 . The traces shown are obtained by numerical integration of model equation (1) with parameters as in Fig. 4. In this paper, the model given by Eq. (1) is numerically solved with Euler's technique and a time step of 0.001 . To pick up transients completely, in all simulations presented below both mean interstimulus duration and window length $t_{b}-t_{a}$ are $\approx 2.5$ times larger than that shown here.

antiphase peaks of the distribution $\left\{\phi_{j}\left(t+\tau_{k}\right)\right\}_{k=1, \ldots, l}$ at time $t$ are specifically detected with the time-dependent antiphase CT clustering index of the $j$ th oscillator defined by

$$
\alpha_{j}(t)=\lambda_{j}^{(2)}(t)-\lambda_{j}^{(1)}(t)
$$

[17]. $-1 \leqslant \alpha_{j}(t) \leqslant 1$ is fulfilled for all times $t$, where the two Dirac-like symmetric antiphase peaks are related to $\alpha_{j}(t)$ $=1 . \alpha_{j}$ detects symmetric CT antiphase response clustering which is stimulus locked in time (see, Ref. [17]). Analogously, we introduce the antiphase clustering index of the $n$ : $m$ phase difference by putting

$$
\delta_{n, m}(t)=\sigma_{n, m}^{(2)}(t)-\sigma_{n, m}^{(1)}(t),
$$


where $\delta_{n, m}(t)$ detects two symmetric antiphase peaks of the distribution $\left\{\varphi_{n, m}\left(t+\tau_{k}\right)\right\}_{k=1, \ldots, l}$.

(iv) Three equally spaced peaks of the distribution $\left\{\phi_{j}(t\right.$ $\left.\left.+\tau_{k}\right)\right\}_{k=1, \ldots, l}$ at time $t$ correspond to large $\lambda_{j}^{(3)}(t)$ and small $\lambda_{j}^{(\nu)}(t)$ for $\nu=1,2$.

$\lambda_{j}^{(\nu)}$ and $\sigma_{n, m}^{(\nu)}$ are the modulus of the $\nu$ th Fourier mode of the CT distributions from Eq. (7) (see, Ref. [5]). Similar to Eq. (8), $Z_{\nu}(t)=l^{-1} \sum_{k=1}^{l} \exp \left[i \nu \xi_{k}(t)\right]$ is used to detect $\nu$ equally spaced phase-locked clusters in a population of $l$ oscillators with phases $\xi_{1}, \ldots, \xi_{l}[5,18,25]$.

In addition to the indices defined by Eqs. (8)-(11), we use indices based on the Shannon entropy in order to quantify the deviation of the distributions $\left\{\phi_{j}\left(t+\tau_{k}\right)\right\}_{k=1, \ldots, l}$ and $\left\{\varphi_{n, m}\left(t+\tau_{k}\right)\right\}_{k=1, \ldots, l}$ from a uniform one. Accordingly, the time-dependent entropy based stimulus-locking index $\mu_{j}(t)$ of $\phi_{j}$ reads

$$
\mu_{j}(t)=\frac{S_{\max }-S_{j}(t)}{S_{\max }},
$$

where $S_{j}(t)=-\sum_{i=1}^{N} p_{i} \ln p_{i}$ is the entropy of the distribution $\left\{\phi_{j}\left(t+\tau_{k}\right)\right\}_{k=1, \ldots, l}$ at time $t$, and $p_{i}$ denotes the relative frequency of finding $\phi_{j}\left(t+\tau_{k}\right)$ within the $i$ th bin. $S_{\max }$ $=\ln N$ is the entropy of a uniform distribution, where $N$ $=\exp [0.626+0.4 \ln (l-1)]$ is the optimal number of bins, and $l$ is the number of stimuli administered [19]. $0 \leqslant \mu_{j}(t)$ $\leqslant 1$ holds for all $t$, where $\mu_{j}(t)=0$ corresponds to a uniform distribution (no stimulus locking) at time $t$, whereas $\mu_{j}(t)$ $=1$ corresponds to a Dirac-like distribution (perfect stimulus locking) at time $t$.

Analogously, the time-dependent entropy based $n: m$ stimulus-locking index $\rho_{n, m}(t)$ of $\varphi_{n, m}$ is given by

$$
\rho_{n, m}(t)=\frac{S_{\max }-S_{n, m}(t)}{S_{\max }},
$$

where $S_{n, m}(t)$ is the entropy of the distribution $\left\{\varphi_{n, m}(t\right.$ $\left.\left.+\tau_{k}\right)\right\}_{k=1, \ldots, l}$ at time $t$. The entropy based index for $\varphi_{n, m}$ from Eq. (13) has been introduced in Ref. [5], whereas the entropy based index for $\phi_{j}$ from Eq. (12) is introduced here.

We use the first and the 99th percentile of the prestimulus distributions of the locking indices $\left\{\lambda_{j}^{(\nu)}(t)\right\}_{t \in\left[t_{a}, 0[\right.}$, $\left\{\sigma_{n, m}^{(\nu)}(t)\right\}_{t \in\left[t_{a}, 0[\right.}, \quad\left\{\mu_{j}(t)\right\}_{t \in\left[t_{a}, 0[\right.}$, and $\left\{\rho_{n, m}(t)\right\}_{t \in\left[t_{a}, 0[\right.}$ as confidence levels in order to determine whether a stimulus causes a significant increase or decrease of the corresponding locking index. For example, an increase or a decrease of the stimulus locking of $\phi_{j}$ at time $t$ is considered significant, provided $\lambda_{j}^{(1)}(t)$ is greater than the 99th or smaller than the first percentile of $\left\{\lambda_{j}^{(1)}(t)\right\}_{t \in\left[t_{a}, 0[\right.}$, respectively. Significant stimulus-locked in-phase synchronization or desynchronization at time $t$ occurs, provided $\sigma_{n, m}^{(1)}(t)$ exceeds the 99th or falls below the first percentile of $\left\{\sigma_{n, m}^{(1)}(t)\right\}_{t \in\left[t_{a}, 0[\right.}$. The differences between the listed indices will be explained below. In this paper, we restrict ourselves to the simplest case with $n=m=1$. The stimulus-locked dynamics of the $n: m$ phase difference (with $n$ and/or $m \neq 1$ ) has to be taken into account, e.g., if the oscillators interact via an $n: m$ coupling consisting of terms such as $\sin \left(n \psi_{j}-m \psi_{k}\right)$.

\section{MOTION IN A DOUBLE-WELL POTENTIAL}

Let us consider the dynamics of model equation (1) without noise by setting $X_{j}$ from Eq. (2) equal to zero. With Eqs. (5) and (1) we immediately obtain the evolution equation of the phase difference $\varphi_{1,1}$ as

$$
\dot{\varphi}_{1,1}=\frac{\mu}{2 \pi}-\frac{K}{\pi} \sin \left(2 \pi \varphi_{1,1}\right)+F(t),
$$

which we cast into the form

$$
\dot{\varphi}_{1,1}=G\left(\varphi_{1,1}\right)+F(t) .
$$

The random forces $F(t)=F_{1}(t)-F_{2}(t)$ are the Gaussian white noise fulfilling $\langle F(t)\rangle=0$ and $\langle F(t) \quad F(\tilde{t})\rangle=D \delta(t$ $-\tilde{t}) / \pi$ with constant noise amplitude $D / \pi . G\left(\varphi_{1,1}\right)$ is a short form for the deterministic terms of the right-hand side of Eq. (14).

First, we focus on the behavior occurring without noise, i.e., for $D=0$. In this case, the dynamics is governed by a potential

$$
V\left(\varphi_{1,1}\right)=-\int_{c}^{\varphi_{1,1}} G(\xi) d \xi
$$

with constant $c$, where

$$
\dot{\varphi}_{1,1}=-\frac{d V\left(\varphi_{1,1}\right)}{d \varphi_{1,1}} .
$$

A suitable choice of $c$ yields

$$
V\left(\varphi_{1,1}\right)=-\frac{\gamma}{2 \pi} \varphi_{1,1}-\frac{K}{2 \pi^{2}} \cos \left(2 \pi \varphi_{1,1}\right) .
$$

The dynamics of Eq. (14) corresponds to an overdamped motion of a particle in the potential $V\left(\varphi_{1,1}\right)$ (Fig. 3). $\varphi_{1,1}$ moves in such a way that $V\left(\varphi_{1,1}\right)$ is minimized, and $\varphi_{1,1}$ stops only when $d V / d \varphi_{1,1}$ vanishes (see Ref. [1] and Chap. 9 in Ref. [24]). The maximum of the potential is an unstable fixed point. We denote the value of $\varphi_{1,1}$ at which $V$ is maximal by $\varphi_{1,1}^{\max }$. For $\varphi_{1,1}=\varphi_{1,1}^{\max }$ a minimal perturbation is sufficient to make $\varphi_{1,1}$ move either to the right or to the left minimum of $V$. The particle relaxes into the right or left minimum depending on whether $\varphi_{1,1}$ is greater or less than $\varphi_{1,1}^{\max }$, respectively.

Studying the poststimulus dynamics of $\varphi_{1,1}$, thus, means considering an initial condition problem of Eq. (14): The stimulus puts $\varphi_{1,1}$ on a particular value, which is $\varphi_{1,1}$ 's initial value of the poststimulus period. Starting at that initial value, $\varphi_{1,1}$ relaxes towards a stable state. By placing $\varphi_{1,1}$ to the right or to the left of $\varphi_{1,1}^{\max }$, the stimulus completely determines whether $\varphi_{1,1}$ moves to the right or to the left minimum.

When noise is added, i.e., for $D>0$, the situation changes. We are no longer able to predict the trajectory of the particle. Rather we can only describe its dynamics in a probabilistic sense. For example, by means of a FokkerPlanck equation we can determine the time course of the 


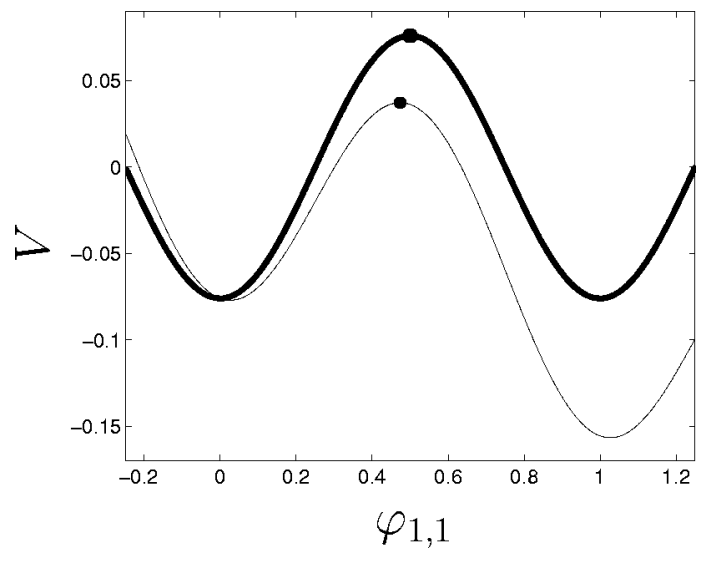

FIG. 3. Potential $V$ from Eq. (18) is plotted for two different values of the detuning $\gamma$ : $\gamma=0$ (thick line), $\gamma=0.5$ (thin line). Local maxima of $V$ are indicated by dots, respectively.

probability density $p\left(\varphi_{1,1}, t\right)$, where $p\left(\varphi_{1,1}, t\right) d \varphi_{1,1}$ gives us the probability of finding $\varphi_{1,1}$ in the interval $\left[\varphi_{1,1}, \varphi_{1,1}\right.$ $\left.+d \varphi_{1,1}\right]$ (see Ref. [1] and Chap. 9 in Ref. [24]). The impact of noise after stimulation is obvious: The overdamped motion of the particle is perturbed by random forces and behaves in a way that has been studied in detail in the context of diffusion in a double-well potential (see, Chap. 9 in Ref. [24]). The dynamics for $t \rightarrow \infty$ is no longer totally determined by the initial state, which means that the division into three different scenarios (i.e., staying at the unstable maximum or moving into the right or left minimum) is no longer valid. For sufficiently large noise amplitude the particle may end up in the right well although it started left from $\varphi_{1,1}^{\max }$ and vice versa.

\section{TRANSIENT RESPONSE CLUSTERING, SYNCHRONIZATION, AND DESYNCHRONIZATION}

We can place the particle on top of the potential $V$ by simultaneously delivering two stimuli with

$$
S_{1}\left(\psi_{1}\right)=I \cos \psi_{1}, \quad S_{2}\left(\psi_{2}\right)=I \cos \left(\psi_{2}+\pi\right),
$$

where both stimuli have identical onsets and offsets $\left(t_{\text {del }}\right.$ $=0$ in Fig. 1). For $I$ large enough compared to the coupling strength $K$, the first stimulus resets $\phi_{1}$ to $\phi^{\text {stat }}$, whereas the second stimulus resets $\phi_{2}$ to $\phi^{\text {stat }}+\pi[16,17]$. In general, such an antiphase reset can be achieved with stimuli of first order, i.e., stimuli containing only terms with $\cos (\psi)$ and $\sin (\psi)$, only provided the phase difference of the arguments of the two stimuli is sufficiently close to $\pi$, such as $S_{1}\left(\psi_{1}\right.$ $+\theta)=I \cos \left(\psi_{1}+\theta\right)$ and $S_{2}\left(\psi_{2}\right)=I \cos \left(\psi_{2}+\theta+\pi\right)$ with constant $\theta[16,17]$.

The question now is, whether we can compensate a phase shift of $\pi$ in the arguments of $S_{1}$ and $S_{2}$ by a delay $t_{\text {del }}$ corresponding to half a period of the oscillators. Put otherwise: Can an antiphase reset be achieved with two stimuli of first order

$$
S_{1}\left(\psi_{1}\right)=I \cos \psi_{1}, \quad S_{2}\left(\psi_{2}\right)=I \cos \left(\psi_{2}\right)
$$

administered with a delay of half a mean period of the oscillators: $t_{\mathrm{del}}=T / 2=\pi / \omega$ in Fig. 1 ?

We assume the coupling to be strong enough compared to the noise amplitude $D$, so that without stimulation the two oscillators spontaneously synchronize in phase [Figs. 4(g) and 4(h)]. The stimulation intensity $I$ is assumed to be large compared to the coupling strength $K$ and to the noise amplitude $D(K \ll I, D \ll I)$. The two stimuli may have different intensity parameters, what matters is that the intensity parameters are large with respect to $K$ and $D$. This guarantees that the two strong stimuli quickly reset the oscillators: When the first stimulus is over, $\phi_{1}$ has been shifted close to $\phi^{\text {stat }} \approx 0.36$ [Fig. 4(a)]. Likewise, after the offset of the second stimulus $\phi_{2}$ has also been reset to $\phi^{\text {stat }} \approx 0.36$ [Fig. 4(e)]. $t_{\text {del }}=T / 2$ is the delay between the offsets of the two stimuli. Therefore, after the offset of the first stimulus, oscillator 1 runs through half a period until stimulation of oscillator 2 is over, too. Consequently, at the end of the stimulation, in total an antiphase reset is achieved, i.e., $\varphi_{1,1}$ is set to 0.5 [Fig. $4(\mathrm{~g})]$.

The reset of the oscillators' phases is reflected by an increase of the locking index $\lambda_{j}^{(1)}$ [Fig. 4(b)]. Due to this reset the oscillators undergo a transition from an in-phase synchronization to a particularly strong antiphase synchronization via a transient desynchronization in between [Figs. 4(g) and 4(h)]. After the stimulation, both oscillators relax back to the same in-phase synchronization as before stimulation. During this relaxation they pass through a desynchronization that lasts longer than the desynchronization during stimulation since $K \ll I$. In the course of the desynchronization during and after stimulation the trajectories of $\varphi_{1,1}$ form two "branches" that converge to and diverge from $\varphi_{1,1}=0.5$ [Fig. 4(g)]. The two branches occur for the following reason. When the particle (meaning $\varphi_{1,1}$ ) is placed on top of the maximum of the potential $V$ from Fig. 3, it moves down to either the left or the right minimum. Note, in the simulation shown in Fig. 4 we have nonvanishing noise. The antiphase position of the two branches of the trajectories of $\varphi_{1,1}$ coincides with a local maximum of $\sigma_{1,1}^{(2)}$ from Eq. (9) [Fig. 4(i)], a local minimum of $\sigma_{1,1}^{(1)}$ [Fig. 4(h)], and, thus, a local maximum of $\delta_{1,1}$ from Eq. (11) [Fig. 4(j)].

After the stimulation the trajectories of both $\phi_{1}$ and $\phi_{2}$ form two antiphase clusters across trials, respectively. This shows up in the locking indices from Eqs. (8) and (10): While $\lambda_{j}^{(1)}$ relaxes to zero, $\lambda_{j}^{(2)}$ reincreases [Figs. 4(b) and 4(c)]. Accordingly, also $\alpha_{j}$ reincreases and even exceeds its 99th prestimulus percentile [Figs. 4(d) and 4(f)]. This indicates that the distribution $\left\{\phi_{j}\left(t_{1}+\tau_{k}\right)\right\}_{k=1, \ldots, l}$ has two antiphase peaks [Figs. 4(a) and 4(e)]. With further increase in time the clustering of $\phi_{j}$ 's trajectories vanishes due to the noise.

The intimate relationship between the branching of $\varphi_{1,1}$ and the antiphase CT response clustering of $\phi_{j}$ has been studied in detail for an antiphase reset achieved with simultaneously delivered stimuli with phase shift of $\pi$ as defined by Eq. (19) $[16,17]$ : In those trials when $\varphi_{1,1}$ runs through the upper or the lower branch, $\phi_{j}$ predominantly belongs to either one of its clusters, respectively. This relationship holds 

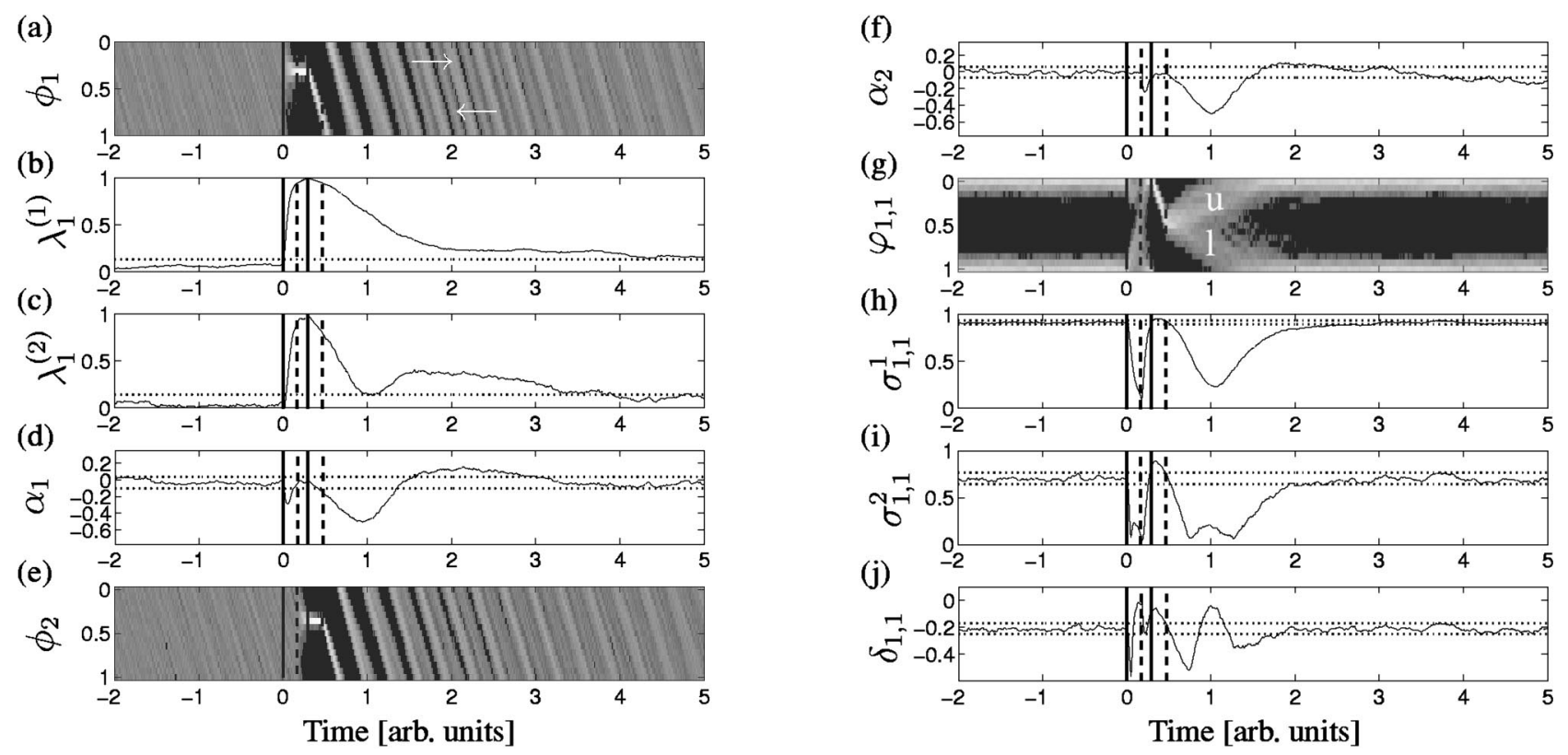

FIG. 4. Strong stimuli $S_{1}\left(\psi_{1}\right)=I \cos \psi_{1}$ and $S_{2}\left(\psi_{2}\right)=I \cos \psi_{2}$ from Eq. (1) administered with a delay of half a mean period of the oscillators $\left(t_{\mathrm{del}}=T / 2=\pi / \omega\right)$ cause an antiphase reset. CT distributions from Eq. (7) are shown as time-dependent histograms of $\phi_{j}$ and $\varphi_{1,1}$ calculated across trials for each time $t$ relative to stimulus onset within the time window $\left[t_{a}, t_{b}\right],\left\{\phi_{1}\left(t+\tau_{k}\right)\right\}_{k=1, \ldots, l}$ in $(\mathrm{a}),\left\{\phi_{2}(t\right.$ $\left.\left.+\tau_{k}\right)\right\}_{k=1, \ldots, l}$ in (e), $\left\{\varphi_{1,1}\left(t+\tau_{k}\right)\right\}_{k=1, \ldots, l}$ in (g) ( 0 is black and maximal values are white). Horizontal arrows indicate antiphase peaks of $\left\{\phi_{1}\left(t+\tau_{k}\right)\right\}_{k=1, \ldots, l}$ at $t=2$ in (a). $u$ and $l$ indicate upper and lower branch of trajectories in (g). Locking indices from Eqs. (8)-(11): $\lambda_{1}^{(1)}$ in (b), $\lambda_{1}^{(2)}$ in (c), $\alpha_{1}$ in (d), $\alpha_{2}$ in (f), $\sigma_{1,1}^{(1)}$ in (h), $\sigma_{1,1}^{(2)}$ in (i), and $\delta_{1,1}$ in (j). $\lambda_{2}^{(1)}$ and $\lambda_{2}^{(2)}$ are very similar to $\lambda_{1}^{(1)}$ and $\lambda_{1}^{(2)}$, respectively. As in Fig. 2, onset (at $t=0$ ) and offset of the stimulus of oscillator 1 are indicated by solid vertical lines, whereas onset and offset of the stimulus of oscillator 2 are denoted by dashed vertical lines. Prestimulus interval, $t<0$, poststimulus interval, $t>0$. Significance levels, dotted lines in (b) and (c) denote the 99th percentile of the prestimulus distributions $\left\{\lambda_{1}^{(\nu)}(t)\right\}_{t \in[-5,0[}$. In (d), (f), and (h) -(j) upper and lower dotted lines indicate the 99 th and the first percentile of the corresponding prestimulus distribution in the interval $[-5,0[$. Note, only a part of the time window $\left[t_{a}, t_{b}\right]=[-5,5.5]$ is displayed for the sake of clarity. Parameters of Eq. (1): $K=1.5, \omega=6 \pi, \gamma=0.04, D=0.5, I$ $=30, t_{\text {win }}=11$ [see, Eq. (6)], stimulus duration $=0.3$, number of stimuli $l=200$. Results are stable with respect to variations of $l$ between 50 and 2000 and more.

also in the case of an antiphase reset achieved with stimuli administered at different times. In our case this means that in those trials when $\varphi_{1,1}$ runs through the upper branch [denoted by $u$ in Fig. 4(g)], the corresponding $\phi_{1}$ is in the upper peak of its CT distribution [marked by the upper arrow in Fig. 4(a)]. By the same token, in those trials with $\varphi_{1,1}$ running through the lower branch $[l$ in Fig. $4(\mathrm{~g})], \phi_{1}$ is contained in the lower peak of its CT distribution [lower arrow in Fig. 4(a)]. We shall come back to this point below.

\section{IMPACT OF THE DELAY BETWEEN THE TWO STIMULI}

To demonstrate the impact of the type of reset on the poststimulus dynamics we vary the delay $t_{\text {del }}$ between the two stimuli (see Fig. 1), while the stimuli $S_{1}$ and $S_{2}$ are kept fixed and are given by Eq. (20). $t_{\text {del }}$ is varied between 0 and $3 T$, where $T=2 \pi / \omega$ is the mean period of the oscillators. For $t_{\text {del }}$ close to $0.5 T, 1.5 T$, and $2.5 T$, an antiphase reset is achieved, which leads to a CT response clustering with its typical signature described in the preceding section: $\lambda_{j}^{(1)}$ from Eq. (8) quickly relaxes to zero [Figs. 5(b) and 5(h)], whereas $\lambda_{j}^{(2)}$ reincreases [Fig. 5(e)], so that also the antiphase CT clustering index $\alpha_{j}$ of both oscillators from Eq. (10) reincreases and displays a distinct transient lasting over more than 20 cycles [Figs. 5(c) and 5(i)]. The corresponding antiphase branching of $\varphi_{1,1}$ 's trajectories shows up as local minimum of $\sigma_{1,1}^{(1)}$ from Eq. (9) [Fig. 5(f)], combined with a local maximum of the antiphase clustering index $\delta_{1,1}$ of the $n: m$ phase difference from Eq. (11) [Fig. 5(k)].

In contrast, when no antiphase reset is performed, i.e., when $t_{\text {del }}$ is not close to $0.5 T, 1.5 T$, and $2.5 T$, the CT response clustering does not occur. In this case, both $\lambda_{j}^{(1)}$ and $\lambda_{j}^{(2)}$ from Eq. (8) relax to zero [Figs. 5(b), 5(e), and 5(h)], without any reincrease of $\lambda_{j}^{(2)}$ [Fig. 5(e)]. Since $\lambda_{j}^{(1)}$ relaxes more slowly compared to $\lambda_{j}^{(2)}$, the antiphase clustering in$\operatorname{dex} \alpha_{j}$ from Eq. (10) undergoes a negative transient before it finally tends to zero. A uniform distribution is connected with $\alpha_{j}=0$.

Also for greater delays, such as $t_{\text {del }}$ close to $3.5 T, 4.5 T$, $5.5 T$ etc., the $\mathrm{CT}$ response clustering occurs. Though less pronounced, the CT response clustering can even be observed for values of the delay up to $t_{\mathrm{del}}=9.5 T$ (not shown, in order to avoid a packed figure).

Figure 5 demonstrates the differences between the two sorts of locking indices, one being designed for detecting specific features, like one peak or two antiphase peaks, of the CT distributions [Eqs. (8)-(11)], the other being based on the Shannon entropy [Eqs. (12) and (13)]. Remarkably, the Sh- 

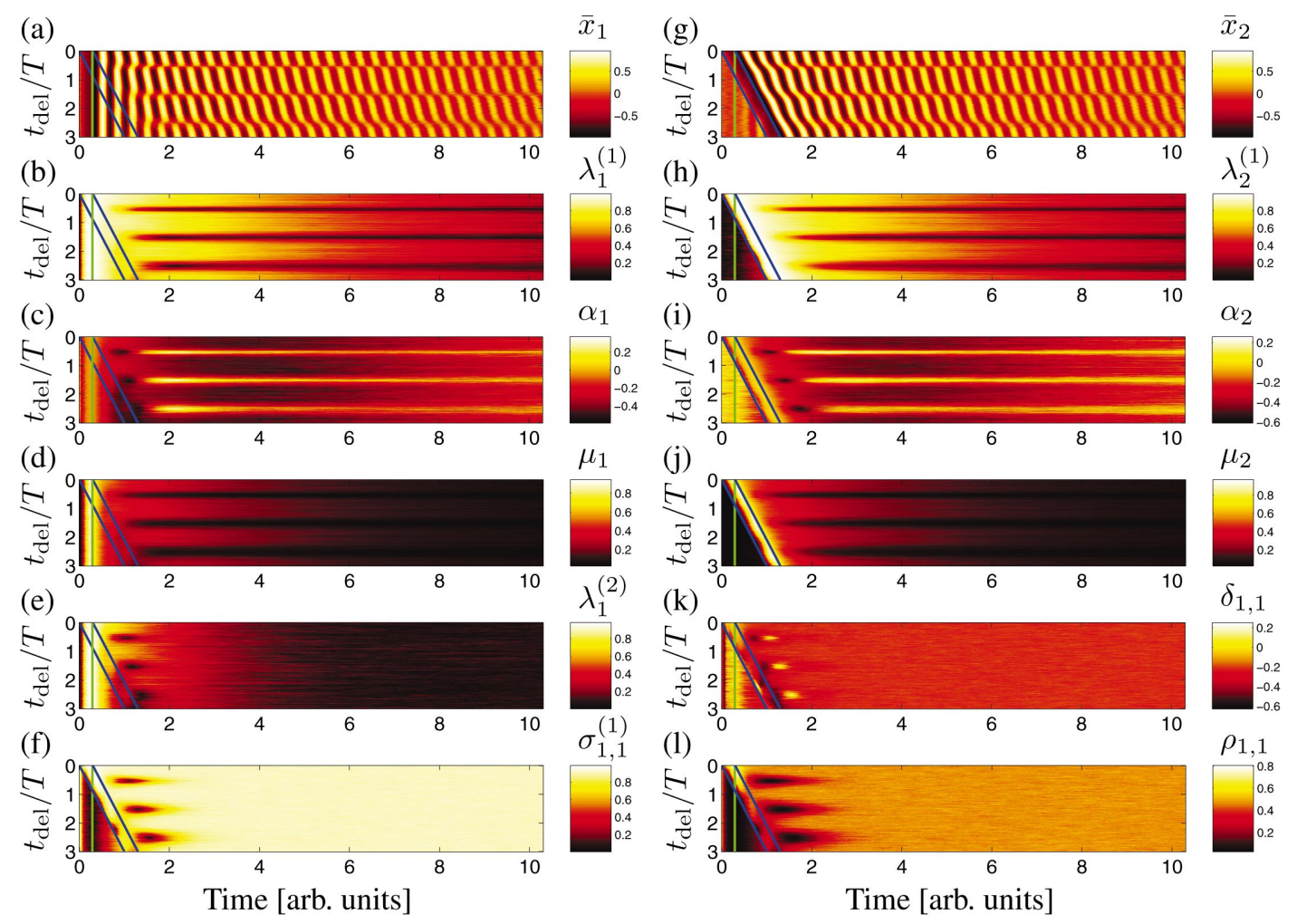

FIG. 5. (Color) The delay $t_{\text {del }}$ between the stimuli $S_{1}\left(\psi_{1}\right)=I \cos \psi_{1}$ and $S_{2}\left(\psi_{2}\right)=I \cos \psi_{2}$ from Fig. 4 is varied between 0 and $3 T$, where $T=2 \pi / \omega$ is the mean period of the oscillators (compare Fig. 1). Except for $t_{\text {del }}$, all other parameters are as in Fig. 4. Onset (at $t=0$ ) and offset (indicated by vertical green line) of oscillator 1 are kept fixed, whereas onset (left sloping blue line) and offset (right sloping blue line) of oscillator 2 are shifted according to $t_{\mathrm{del}}$. CT averaged signals from Eq. (21) and locking indices from Eqs. (8)-(13), $\bar{x}_{1}$ in (a), $\lambda_{1}^{(1)}$ in (b), $\alpha_{1}$ in (c), $\mu_{1}$ in (d), $\lambda_{1}^{(2)}$ in (e), $\sigma_{1,1}^{(1)}$ in (f), $\bar{x}_{2}$ in (g), $\lambda_{2}^{(1)}$ in (h), $\alpha_{2}$ in (i), $\mu_{2}$ in (j), $\delta_{1,1}$ in (k), and $\rho_{1,1}$ in (l). $\lambda_{2}^{(2)}$ is very similar to $\lambda_{1}^{(2)}$.

annon based indices $\mu_{j}$ [Figs. 5(d) and 5(j)] from Eq. (12) behave very similar to $\lambda_{j}^{(1)}$ [Figs. 5(b) and 5(h)] from Eq. (8). In particular, $\mu_{j}$ does not detect antiphase peaks of the CT distribution $\left\{\phi_{j}\left(t+\tau_{k}\right)\right\}_{k=1, \ldots, l}$ in a sufficient way: There is no re-increase of $\mu_{j}$ as observed for $\lambda_{j}^{(2)}$ [Figs. 5(d) and 5(e)]. Consequently, $\mu_{j}$ does not capture the antiphase response clustering. Likewise, the Shannon entropy based index $\rho_{1,1}$ [Fig. 5(1)] from Eq. (13) does not detect the antiphase branching of $\varphi_{1,1}$ 's trajectories. In contrast, the latter does not escape detection when the indices $\sigma_{1,1}^{(1)}$ and $\delta_{1,1}$ [Figs. 5(f) and 5(k)] Eqs. (9) and (11) are used.

In summary, indices $\mu_{j}$ and $\rho_{1,1}$, which are based on the Shannon entropy, are not sensitive enough to pick up the antiphase CT response clustering and the CT antiphase branching of $\varphi_{1,1}$.

\section{COMPARISON WITH THE STANDARD CROSS-TRIAL ANALYSIS}

In this section we apply univariate and bivariate standard cross-trial analysis techniques to the simulations shown in Figs. 4 and 5.

\section{A. Cross-trial averaging}

The gold standard in neuroscience and medicine for extracting stimulus-locked responses of an oscillator is CT averaging relative to stimulus onset $[14,15]$. To compute a CT average of signal $x_{j}$ from Eq. (3) of the $j$ th phase oscillator, we use the stimulus onset $\tau_{k}$ as trigger. With this, the crosstrial averaged signal of the $j$ th oscillator reads

$$
\bar{x}_{j}(t)=\frac{1}{l} \sum_{k=1}^{l} x_{j}\left(\tau_{k}+t\right) .
$$

The assumption behind the triggered averaging is that a response $x_{j}$ can be decomposed into a stereotypical evoked response $e_{j}$, which follows the stimulus with a constant delay, and additive Gaussian noise $\xi_{j}$, so that

$$
x_{j}\left(\tau_{k}+t\right)=e_{j}(t)+\xi_{j}\left(\tau_{k}+t\right)
$$

holds $[14,15]$. In such a case averaging improves the signalto-noise ratio by $\sqrt{l}$, where the number of responses $l$ typically equals $20-300$, and $\bar{x}_{j}(t) \rightarrow e_{j}(t)$ for $l \rightarrow \infty[14,15]$.

Obviously, the assumption from Eq. (22) is violated by the stochastic model given by Eq. (1), because the oscillators perform an ongoing oscillation, the stimulation effect depends on the phase of the oscillator, and the model's noise is not simply added to the signal $x_{j}$, but is inherent in the dynamics. Anyhow, the simple model defined by Eq. (1) shares basic features with stimulated brain activity.

(i) Ongoing oscillations abound in the brain [20].

(ii) Evoked responses result from reorganizing part of these ongoing oscillations, especially by resetting their phase dynamics $[21,22]$. For example, auditory stimuli cause an 
(a)

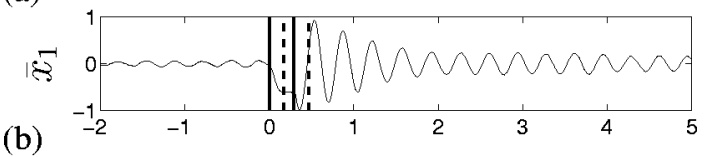

(b)
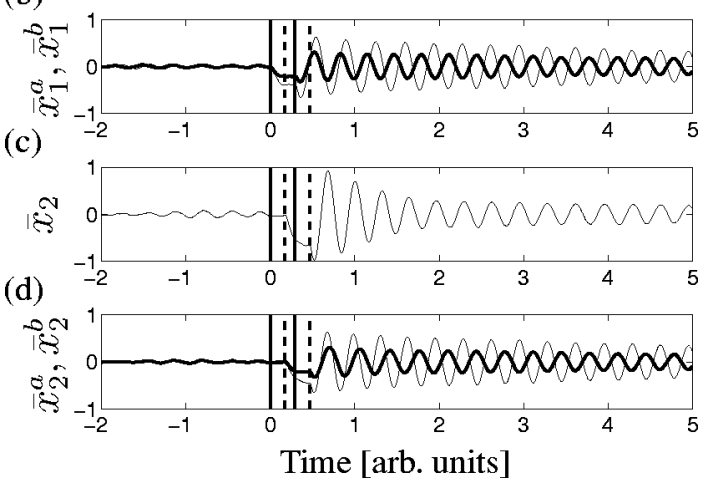

FIG. 6. Time course of the CT averaged responses $\bar{x}_{1}$ (a) and $\bar{x}_{2}$ (c) from Eq. (21) belonging to the simulation from Fig. 4 (and Fig. 5 for $t_{\text {del }}=T / 2$ ). According to Eq. (23) selectively averaged CT responses $\bar{x}_{j}^{a}$ [(b) and (d), thick line $]$ and $\bar{x}_{j}^{b}$ [(b) and (d), thin line $]$ are computed for the trajectories of $\varphi_{1,1}$ running through the upper and the lower branches of Fig. 4(g), respectively.

evoked electroencephalography (EEG) response mainly by changing the phases (but not the amplitudes) of the Fourier spectrum of the spontaneous, pre-stimulus neuronal oscillatory activity [21].

(iii) Noise is inevitably inherent in neuronal action [23]. Therefore, also in neuroscience it is highly questionable whether the averaging assumption is justified. Nevertheless, averaging is typically used for noise reduction of biological signals, such as EEG $[6,14]$ and magnetoencephalography (MEG) signals $[15,20]$, as well as local field potentials (LFP) [4].

The effect of the antiphase CT response clustering of $\phi_{1}$ and $\phi_{2}$ on the CT averaged responses $\bar{x}_{1}$ and $\bar{x}_{2}$ is significant. In the prestimulus region, $\bar{x}_{1}$ and $\bar{x}_{2}$ vanish because of the randomized stimulus administration [Figs. 6(a) and 6(c)]. The stimuli reset $\phi_{1}$ and $\phi_{2}$, and hence in the course of the stimulation $\bar{x}_{1}$ and $\bar{x}_{2}$ approach a constant value [Figs. 5(a) and 5(g) and 6(a) and 6(c)]. In case of an antiphase reset, i.e., for $t_{\text {del }}$ close to $0.5 T, 1.5 T$, and $2.5 T$, after stimulation $\bar{x}_{1}$ and $\bar{x}_{2}$ display strongly damped oscillations. In contrast, without an antiphase reset the oscillations of $\bar{x}_{1}$ and $\bar{x}_{2}$ relax slowly [Figs. 5(a) and 5(g)]. The strong damping of the CT averaged responses $\bar{x}_{1}$ and $\bar{x}_{2}$ is caused by the antiphase CT response clustering. To see this, let us focus on the situation for $t_{\mathrm{del}}=0.5$ in more detail.

We demonstrate how the antiphase CT response clustering of $\phi_{j}$ is connected with the antiphase CT branching of $\varphi_{1,1}$ 's trajectories. The trajectories of $\varphi_{1,1}$ form an upper and a lower branch [denoted by $u$ and $l$ in Fig. 4(g)]. The two branches are in antiphase position at time $t_{0}=1.0$, when $\sigma_{1,1}^{(1)}$ is locally minimal, whereas $\sigma_{1,1}^{(2)}$ as well as $\delta_{1,1}$ are locally maximal. Let us average selectively over those responses $x_{j}$ that belong to the two different branches. We denote the CT averaged signals of those trials where $\varphi_{1,1}$ runs through the upper and the lower branch by $\bar{x}_{j}^{a}$ and $\bar{x}_{j}^{b}$, respectively:

$$
\bar{x}_{j}^{a}(t)=\frac{1}{l} \sum_{k \in U} x_{j}\left(\tau_{k}+t\right), \quad \bar{x}_{j}^{b}(t)=\frac{1}{l} \sum_{k \in L} x_{j}\left(\tau_{k}+t\right),
$$

where $U$ and $L$ denote the subsets of trials for which $\varphi_{1,1}\left(t_{0}+\tau_{k}\right)$ is in the upper and lower branches: $\varphi_{1,1}\left(t_{0}\right.$ $\left.+\tau_{k}\right) \leqslant 0.5$ for $k \in U$ and $\varphi_{1,1}\left(t_{0}+\tau_{k}\right)>0.5$ for $k \in L$. (Because of the graphics program used, in Fig. $4(\mathrm{~g})$ the $y$ axis runs downwards, so that the values of $\varphi_{1,1}$ in the upper branch are smaller than those in the lower branch.)

At the end of the stimulation, $\bar{x}_{j}^{a}$ and $\bar{x}_{j}^{b}$ are in phase, but within only four poststimulus cycles the phase relationship between $\bar{x}_{j}^{a}$ and $\bar{x}_{j}^{b}$ turns from in phase into antiphase [Figs. 6(b) and 6(d)]. According to Eq. (23), $\bar{x}_{j}^{a}$ and $\bar{x}_{j}^{b}$ are normalized by a factor of $1 / l$, so that $\bar{x}_{j}^{a}(t)+\bar{x}_{j}^{b}(t)=\bar{x}_{j}(t)$ for all times $t$. As a further consequence of this normalization, if the majority of trajectories of $\varphi_{1,1}$ run through only one of the branches, the corresponding CT averaged signal is large, whereas the other one is small. This is what we observe in our case: $67 \%$ of the trajectories of $\varphi_{1,1}$ run through the upper branch, and only 33\% run through the lower branch. Therefore, $\bar{x}_{j}^{a}$ has a larger amplitude compared to that of $\bar{x}_{j}^{b}$. Hence, when $\bar{x}_{j}^{a}$ and $\bar{x}_{j}^{b}$ are in antiphase relation, they do not cancel each other out, but $\bar{x}_{j}^{a}$ dominates $\bar{x}_{j}$, so that a lowamplitude oscillation persists.

A phenomenological interpretation of $\bar{x}_{j}$ 's dynamics in the spirit of the evoked response literature $[6,14]$ would be as follows: Before stimulation the oscillators are not active (Fig. 6), the stimulus activates them, and their response quickly decays to a low-amplitude oscillation (for $t_{\text {del }}$ close to $0.5 T, 1.5 T, 2.5 T$, etc.) or their response persists during a long epoch and relaxes only slowly (else) [Figs. 5(a) and $5(\mathrm{~g})$ ]. But according to Eq. (1), the oscillators are permanently active with constant amplitude, irrespective of $t_{\mathrm{del}}$.

In summary, with CT averaging as defined by Eq. (21), it is impossible to distinguish between a mean amplitude decrease, of the single responses and a CT response decorrelation which is, e.g., due to an antiphase CT response clustering. Consequently, the CT averaging may lead to severe artifacts.

\section{B. Cross-trial standard deviation}

To estimate whether the poststimulus signals of the oscillators are stereotypical, we determine the standard deviation across trials at each time $t$ relative to stimulus onset with the cross-trial standard deviation of the $j$ th oscillator defined by

$$
\varrho_{j}(t)=\sqrt{\frac{1}{l-1} \sum_{k=1}^{l}\left[x_{j}\left(\tau_{k}+t\right)-\bar{x}_{j}(t)\right]^{2}},
$$

with the CT averaged signal $\bar{x}_{j}$ from Eq. (21), and $x_{j}(t)$ $=\cos \left[2 \pi \phi_{j}(t)\right]$ according to Eqs. (3) and (4). We would 
expect $\varrho_{j}(t)$ to be small when the signals $x_{j}\left(\tau_{k}+t\right)$ are perfectly stimulus locked at time $t$, and large when there is no stimulus locking.

To demonstrate important features of the CT standard deviation (and of another CT analysis below) we introduce noise-free, idealized responses. The latter are not generated by the model given by Eq. (1), but are simply defined in order to serve as test data. Consider an ensemble of responses of both oscillators defined by

$$
\begin{gathered}
\phi_{1}\left(t+\tau_{k}\right)=\left[t+\varepsilon \xi_{1, k}\right] \bmod 1, \\
\phi_{2}\left(t+\tau_{k}\right)=\left[t+\Delta \phi+\varepsilon \xi_{2, k}\right] \bmod 1,
\end{gathered}
$$

for $k=1, \ldots, l$, where $\Delta \phi$ is the constant mean phase difference between the responses of the two oscillators, and $\left\{\xi_{j, k}\right\}_{k=1}^{l}$ is constant and normally distributed with variance 1 for $j=1,2$. We modify the variance of the normal distributions of the responses by varying $\varepsilon$. Both synthetic oscillators have an identical frequency and a period $T=1$. Note, for each oscillator the CT standard deviation of the phases $\phi_{j}\left(t+\tau_{k}\right)$ of all responses $k=1, \ldots, l$ is constant in time. Furthermore, the phase difference $\varphi_{1,1}\left(t+\tau_{k}\right)$ of all responses $k=1, \ldots, l$ is constant in time $t$. Hence, quantities measuring the extent of the stimulus locking of the responses of each single oscillator as well as their interdependence have to be constant, too.

This requirement, however, is violated by the $\mathrm{CT}$ standard deviation $\varrho_{j}$ defined by Eq. (24) [Fig. 7(a)]. Only for nearly vanishing variance of the responses, i.e., for $\varepsilon$ close to $0, \varrho_{j}$ is (nearly) constant in time, whereas for larger values of $\varepsilon$, we observe an "artificial" oscillation of $\varrho_{j}$ with a period of $T / 2$, where $T$ is the period of the synthetic oscillators. This oscillation occurs for all values of $\Delta \phi$ from Eq. (26).

Accordingly, when we calculate $\varrho_{j}$ for the simulated data from Fig. 4, we also observe an artificial oscillation [Fig. 7(c)]: Directly after the stimulation $\varrho_{j}$ is close to zero, which, of course, makes sense, since it reflects the reset. But the poststimulus increase of $\varrho_{j}$ is modulated by the artificial oscillation already observed in the synthetic data [Fig. 7(a)].

In summary, the CT standard deviation from Eq. (24) cannot be considered as a reliable measure for stimulus locking of the responses of a single oscillator, since it produces artificial oscillations that are not related to stimulus locked dynamics.

\section{Cross-trial cross correlation}

Let us recall what happens if we detect linear correlations between the two oscillators across trials by applying the cross correlation across trials to the signals of the oscillators at each time $t$ relative to stimulus onset $[16,17]$ :

$$
C(t)=\frac{\sum_{k=1}^{l} x_{1}\left(t+\tau_{k}\right) x_{2}\left(t+\tau_{k}\right)}{\sqrt{\left[\sum_{k=1}^{l} x_{1}^{2}\left(t+\tau_{k}\right)\right]\left[\sum_{k=1}^{l} x_{2}^{2}\left(t+\tau_{k}\right)\right]}}
$$
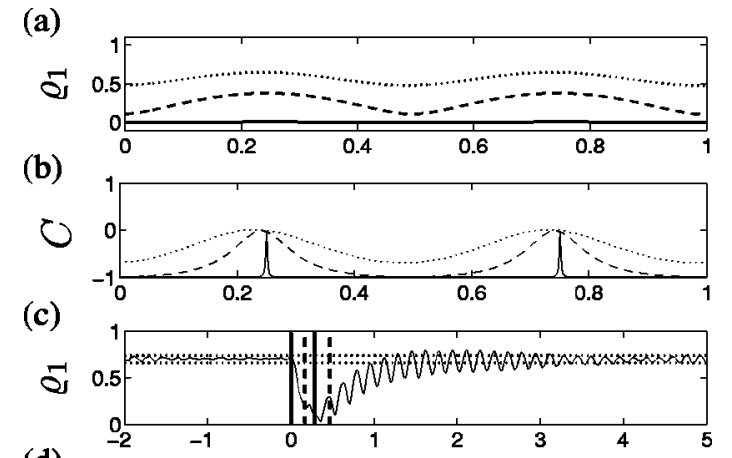

(d)

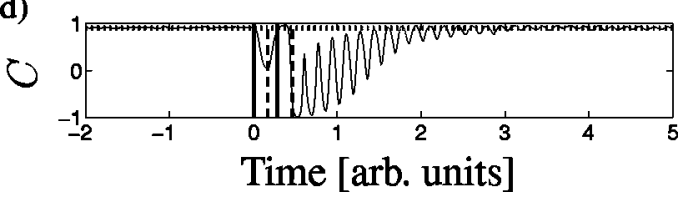

FIG. 7. The CT standard deviation $\varrho_{1}$ from Eq. (24) (a) and CT cross-correlation $C$ from Eq. (27) (b) for synthetic antiphase responses defined by Eqs. (25) and (26), $\phi_{1}\left(t+\tau_{k}\right)=[t$ $\left.+\varepsilon \xi_{1, k}\right] \bmod 1$ and $\phi_{2}\left(t+\tau_{k}\right)=\left[t+\Delta \phi+\varepsilon \xi_{2, k}\right]$ with $\Delta \phi=0.5(k$ $=1, \ldots, l)$. The time interval $[0,1]$ equals one period of the synthetic responses. $\left\{\xi_{j, k}\right\}_{k=1}^{l}$ is constant in time and normally distributed with unit variance for $j=1,2$. The variance of the responses is modified by varying $\varepsilon: \varepsilon=0.01$ (solid line), $\varepsilon=0.4$ (dashed line), and $\varepsilon=1$ (dotted line), so that the overall variance of $\varepsilon\left\{\xi_{j, k}\right\}_{k=1}^{l}$ reads 0.0001 (solid line), 0.16 (dashed line), and 1 (dotted line). For $\varepsilon=0, C$ always equals -1 , except for the times when the signals $x_{j}=\cos \left(2 \pi \phi_{j}\right)$ vanish. A constant scattering of the phases $(\varepsilon>0)$ makes $C$ smoother, so that oscillations with twice the oscillators' frequency occur-although the phase difference $\varphi_{n, m}$ of all pairs of responses is constant. $\varrho_{2}$ is very similar to $\varrho_{1}$. (c) and (d) $\varrho_{1}$ and $C$ are computed for the signals from Fig. 4 (same format as in Fig. 4). Again, $\varrho_{2}$ is very similar to $\varrho_{1}$.

is the cross-trial cross correlation between $x_{1}$ and $x_{2}$ at time $t$, which (to avoid a singularity) by definition is set to zero if all responses of $x_{1}$ or $x_{2}$ vanish at time $t$. $C$ is normalized: $-1 \leqslant C(t) \leqslant 1$ for all times $t . C(t)=1$ or -1 if $x_{1}\left(t+\tau_{k}\right)$ $=c x_{2}\left(t+\tau_{k}\right)$ with constant $c>0$ or $<0$ for all $k$ $=1, \ldots, l$.

The CT cross correlation of the synthetic stimulus-locked responses from Eqs. (25) and (26) artificially oscillates with increasing time $t$, i.e., with increasing phases $\phi_{j}$ although the phase difference $\varphi_{n, m}$ remains constant [Fig. 7(b)]. These oscillations occur for all values of the phase difference $\Delta \phi$ $[16,17]$.

Correspondingly, artificial oscillations are also observed when the CT cross correlation is applied to the simulated data from Fig. 4. Prior to stimulation the oscillators are synchronized with phases that are not stimulus locked due to the randomized stimulus administration according to Eq. (6). $C$ is nearly constantly close to 1 [Fig. $7(\mathrm{~d})]$. The stimulus causes an antiphase reset, so that $C$ is set close to -1 . While the stimulus-locked poststimulus responses resynchronize, $C$ oscillates with twice the oscillators' eigenfrequency [as in Fig. 7(b)]. Correspondingly, the CT cross correlation defined by Eq. (27) is not an appropriate measure for stimuluslocked synchronization and desynchronization.

Also, a modification of Eq. (27) performed to avoid sin- 
gular behavior in case all responses of $x_{1}$ or $x_{2}$ vanish at time $t$ does not help to get rid of the oscillatory artifacts: A crosstrial sign cross correlation was introduced by $\Gamma(t)$ $=l^{-1} \sum_{k=1}^{l} \operatorname{sgn}\left[x_{1}\left(t+\tau_{k}\right) x_{2}\left(t+\tau_{k}\right)\right]$, where $\operatorname{sgn}(a)=-1,0$ or 1 if $a<0,=0$ or $>0[16,17]$. Although different by definition, the CT cross correlation from Eq. (27) and the CT sign cross correlation $\Gamma(t)$ are very similar, at least when applied to signals stemming from phase oscillators. They do not only depend on the phase difference $\varphi_{n, m}$, but inevitably also on the oscillators' absolute phases $\phi_{j}$. Thus, both measures lead to severe artifacts.

\section{STOCHASTIC RESONANCE OF CT RESPONSE CLUSTERING}

In Sec. IV we already discussed the dynamics of the phase difference $\varphi_{1,1}$ in the absence of noise $(D=0)$. Depending on whether the stimulus places the particle (i.e., $\varphi_{1,1}$ ) to the right or to the left of the maximum of the potential $V$ from Eq. (18) located in $\varphi_{1,1}^{\max }$, the particle moves into the right or left minimum of $V$ (Fig. 3). For $\varphi_{1,1}=\varphi_{1,1}^{\max }$ a minimal perturbation (even numerical noise) is sufficient to make $\varphi_{1,1}$ relax to one of the minima of $V$. This is the case, e.g., for nondetuned oscillators $(\gamma=0)$ subject to a perfect antiphase reset (Fig. 3, thick line). The situation is different if an antiphase reset is performed in detuned oscillators $(\gamma \neq 0$, Fig. 3 , thin line). Since for positive $\gamma$ an antiphase reset places $\varphi_{1,1}$ to the right of $\varphi_{1,1}^{\max }$, the particle will always reach the right minimum of $V$, and there will be no CT branching of the trajectories of $\varphi_{1,1}$.

To study the influence of the noise amplitude on the extent of symmetric antiphase response clustering in detuned oscillators, we use the index of maximal poststimulus antiphase CT clustering of the $j$ th oscillator

$$
\left.\left.\alpha_{j}^{\max }=\max \left\{\alpha_{j}(t) ; t \in\right] t_{\mathrm{E}}, t_{b}\right]\right\}
$$

with $\alpha_{j}$ from Eq. (10). $t_{\mathrm{E}}$ denotes the end of the stimulation, i.e., the offset of the second stimulus (see Fig. 1). Furthermore, to distinguish between the effects of noise during and after stimulation, we determine the fraction of trials with 0 $\leqslant \varphi_{1,1}<0.5$ at time $t$ with

$$
r(t)=\frac{\left[\text { number of trials with } 0 \leqslant \varphi_{1,1}\left(t+\tau_{k}\right)<0.5\right]}{(\text { total number of trials })},
$$

where the total number of trials equals $l$. Note, according to Eq. (5) $\varphi_{1,1}$ is a cyclic variable fulfilling $0 \leqslant \varphi_{1,1} \leqslant 1$ at all times $t . r(t)$ is evaluated at two different times: at time $t_{\mathrm{E}}$ and at time $t_{\max }$, the time at which the maximal CT branching of $\varphi_{1,1}$ 's trajectories occurs. In other words, $t_{\max }$ is the poststimulus time when $\sigma_{1,1}^{(1)}$ from Eq. (9) is minimal. Analogously, we could also define $t_{\max }$ as the poststimulus time when $\delta_{1,1}$ from Eq. (11) is maximal (see Sec. V). More or fewer trajectories run through the upper branch than through the lower branch in Fig. 4(g) (where $0 \leqslant \varphi_{1,1}<0.5$ ), provided $r\left(t_{\max }\right)>0.5$ or $r\left(t_{\max }\right)<0.5$, respectively.
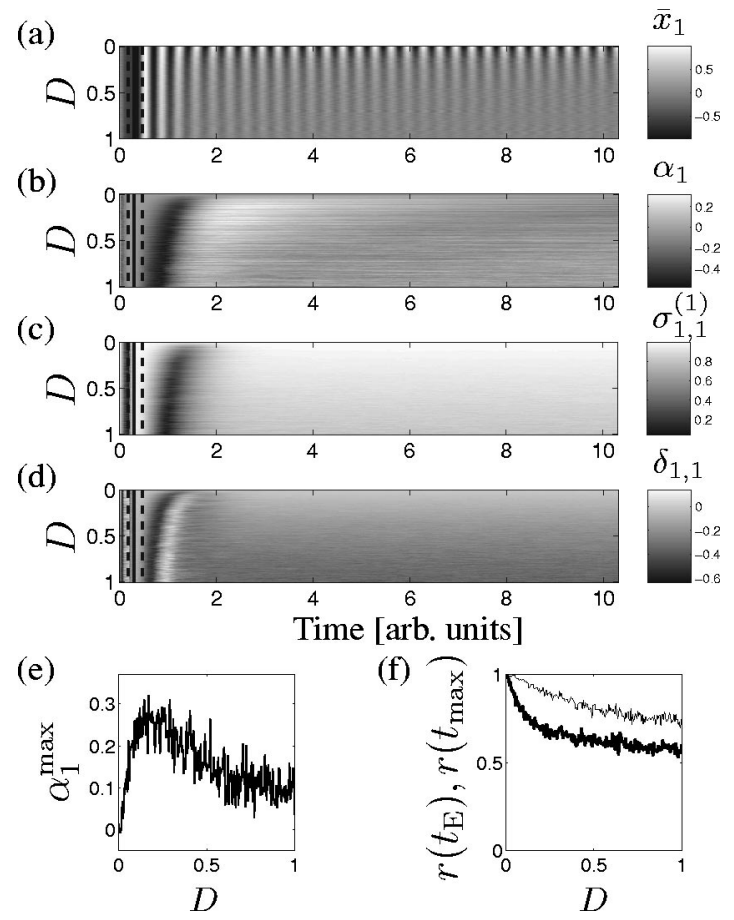

FIG. 8. For the model studied in Fig. 4 the noise amplitude $D$ is varied between 0 and 1 . The number of stimuli reads $l=400$, whereas all other parameters are as in Fig. 4. The time courses of the CT averaged signal $\bar{x}_{1}$ (a) from Eq. (21) and of the locking indices $\alpha_{1}(\mathrm{~b}), \sigma_{1,1}^{(1)}(\mathrm{c})$, and $\delta_{1,1}$ (d), from Eqs. (10), (9), and (11) are displayed. Stochastic resonance is shown with $\alpha_{1}^{\max }(\mathrm{e})$ from Eq. (28). The ratios $r\left(t_{\mathrm{E}}\right)$ (thin line) and $r\left(t_{\max }\right)$ (thick line) from Eq. (29) are plotted in (f), where $t_{\mathrm{E}}$ is the end of the stimulation, i.e., the offset of the second stimulus, and $t_{\max }$ is the time at which the CT response clustering (and thus $\delta_{1,1}$ ) is maximal, i.e., the poststimulus time when $\sigma_{1,1}^{(1)}$ is minimal. $\bar{x}_{2}, \alpha_{2}$, and $\alpha_{2}^{\max }$ are similar to $\bar{x}_{1}, \alpha_{1}$, and $\alpha_{1}^{\max }$, respectively.

We turn to the oscillators shown in Fig. 4. Their detuning $\gamma$ equals 0.04 , and the delay between the first and second stimulus obeys $t_{\mathrm{del}}=0.5 T$. Model equation (1) is now integrated numerically with noise amplitude $D$ varying between 0 and 1 (Fig. 8). For $D=0$ the CT averaged response $\bar{x}_{j}$ relaxes only very slowly [Fig. 8(a)], and no CT response clustering occurs [Fig. 8(b)]. With increasing $D$ we observe a much quicker relaxation of $\bar{x}_{j}$ [Fig. 8(a)], and the antiphase response clustering is reflected by an epoch with positive antiphase CT clustering index $\alpha_{j}$, which is particularly strong for values of $D$, around 0.2 [Fig. 8(b)].

Stochastic resonance of symmetric cross-trial antiphase response clustering is demonstrated by plotting the index of maximal poststimulus antiphase CT clustering $\alpha_{j}^{\max }$ from Eq. (28) over $D$ [Fig. 8(e)]. Without noise, there is no response clustering. For intermediate values of the noise amplitude ( $D$ around 0.2 ) the response clustering is the strongest, whereas with further increase in $D$ the extent of response clustering decreases again. A noise dependence of this kind is a hallmark of stochastic resonance [26].

$r(t)$, i.e., the fraction of trials with $0 \leqslant \varphi_{1,1}<0.5$ at time $t$ defined by Eq. (29), enables us to distinguish between the 
effect of noise during stimulation and the effect after stimulation. Without noise, the stimulus always places the particle to the left of 0.5: $r\left(t_{\mathrm{E}}\right)=1$ [Fig. 8(f)]. Moreover, without noise, the particle always relaxes into the left minimum of $V$ : $r\left(t_{\max }\right)=1$. Thus, the stimulus always places the particle even to the left of the maximum of the potential $V$, which for the detuned oscillators $(\gamma=0.04)$ is slightly shifted to the left: $\varphi_{1,1}^{\max }=0.4980$ (compare Fig. 3). The stimulation does not cause a perfect antiphase reset with $\varphi_{1,1}\left(t_{\mathrm{E}}\right)=0.5$, but a reset with a tendency towards values of $\varphi_{1,1}\left(t_{\mathrm{E}}\right)<0.5$, because both oscillators are coupled. During the period of time when only oscillator 2 is stimulated, i.e., between the offset of the first stimulus and the offset of the second stimulus (Fig. 1), oscillator 1 adapts its phase to the phase of the stimulated oscillator 2.

In the presence of noise, the CT response clustering occurs because of two effects of the random forces.

Effect of noise in the course of the stimulation. Without noise the same stimulus applied to the oscillators in the same dynamical state always moves the particle to the same place in potential $V$. In contrast, as a consequence of the random forces the trajectory of $\varphi_{1,1}$, is no longer predictable. Applying the same stimulus to the same dynamical state several times, leads to a noise-induced scattering of $\varphi_{1,1}\left(t_{\mathrm{E}}\right)$. Hence, $r\left(t_{\mathrm{E}}\right)$ decreases with increasing noise [Fig. 8(f)].

Effect of noise after the stimulation. As already discussed in Sec. IV, for sufficiently large noise amplitude the particle may relax into the right potential well, although it started left from $\varphi_{1,1}^{\max }$ and vice versa. Accordingly, with increasing $D$ the fraction $r\left(t_{\max }\right)$ decreases even quicker compared to $r\left(t_{\mathrm{E}}\right)$ [Fig. 8(f)].

However, with further increasing noise the CT response clustering finally fades away, so that the strongest CT response clustering is observed for intermediate noise amplitude [Fig. 8(e)].

\section{COUPLING-MEDIATED RESONANCE OF CT RESPONSE CLUSTERING}

This section is devoted to the role of the couplings. On one hand, without coupling $(K=0)$ the potential $V$ from Eq. (18) (Fig. 3) is simply a line with a zero slope (for vanishing detuning $\gamma$ ) or a nonzero slope (for $\gamma \neq 0$ ). Hence, without coupling no CT branching of $\varphi_{1,1}$ 's trajectories can occur. On the other hand, in the preceding section we have seen, that when the coupling strength $K$ is much greater than the noise amplitude, an antiphase reset can no longer be achieved by delivering two stimuli with a delay of $t_{\mathrm{del}}=0.5$.

Again, we consider the oscillators shown in Fig. 4. Their detuning $\gamma$ equals 0.04 , and the delay between the first and second stimulus reads $t_{\mathrm{del}}=0.5 T$. Model equation (1) is now solved numerically with noise amplitude $D=0.04$ and coupling strength $K$ varying between 0 and 4 (Fig. 9). For $K$ $=0$, the $\mathrm{CT}$ averaged response $\bar{x}_{j}$ relaxes only very slowly [Fig. 9(a)], and no CT response clustering is observed [Fig. 9(b)]. With increasing $K$ we observe a much quicker relaxation of $\bar{x}_{j}$ [Fig. 9(a)], and a transient epoch with strong antiphase CT response clustering occurs [Fig. 9(b)]. The CT
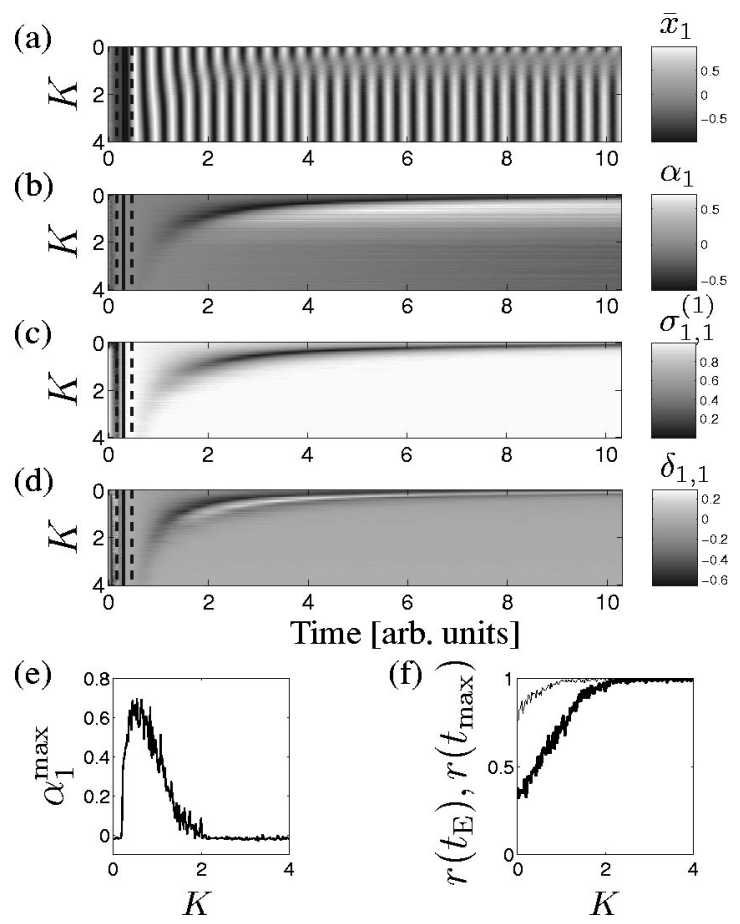

FIG. 9. For the model studied in Fig. 4 the coupling strength $K$ is varied between 0 and 4 . The noise amplitude reads $D=0.04$, whereas all other parameters are as in Fig. 4. The time courses of the CT averaged signal $\bar{x}_{1}$ (a) from Eq. (21) and of the locking indices $\alpha_{1}(\mathrm{~b}), \sigma_{1,1}^{(1)}(\mathrm{c})$, and $\delta_{1,1}(\mathrm{~d})$, from Eqs. (10), (9), and (11) are plotted. Coupling-mediated resonance is demonstrated with $\alpha_{1}^{\max }$ (e) from Eq. (28). The ratios $r\left(t_{\mathrm{E}}\right)$ (thin line) and $r\left(t_{\max }\right)$ (thick line) from Eq. (29) are plotted in (f), where $t_{\mathrm{E}}$ is the end of the stimulation, i.e., the offset of the second pulse, and $t_{\max }$ is the time at which the CT response clustering is maximal. $\bar{x}_{2}, \alpha_{2}$, and $\alpha_{2}^{\max }$ are similar to $\bar{x}_{1}, \alpha_{1}$, and $\alpha_{1}^{\max }$, respectively.

response clustering as assessed with $\alpha_{1}^{\max }$ from Eq. (28) equals zero for $K=0$ and is negative for $K>2$ (tested for $K$ up to 20). In between, $\alpha_{1}^{\max }$ is maximal around $K=0.5$, where it reaches values around 0.7. Accordingly, we observe a distinct resonance behavior.

A similar resonance behavior is observed for a range of values of the noise amplitude. Using, e.g., the fixed noise amplitude $D=0.2$, a value related to maximal stochastic resonance (Fig. 8), the range of coupling-mediated resonance increases towards larger values of the coupling strength, so that $\alpha_{1}^{\max }$ falls below zero only for $K>3.5$.

The fraction $r(t)$ of trials with $0 \leqslant \varphi_{1,1}<0.5$ at time $t$ defined by Eq. (29) tells us more about the interplay of coupling and noise. As in the preceding section, $r$ is evaluated at the end of the stimulation (at $t=t_{\mathrm{E}}$ ) and when the CT branching of $\varphi_{1,1}$ 's trajectories is maximal (at $t=t_{\max }$ ), i.e., when the poststimulus $n: m$ stimulus locking index $\sigma_{1,1}^{(1)}$ from Eq. (9) is minimal [Fig. 9(c)]. Alternatively, we could also identify $t=t_{\max }$ by detecting the maximum of the poststimulus antiphase CT clustering index of the $n: m$ phase difference $\delta_{1,1}$ from Eq. (11) [Fig. 9(d)].

With increasing $K$, more and more trajectories of $\varphi_{1,1}$ are captured in the interval $[0,0.5$ [ at the end of the stimulation, 
at time $t_{\mathrm{E}}$ [Fig. 9(f)]. During the poststimulus transient, noise counteracts this effect by making $\varphi_{1,1}$ diffuse in double-well potential $V$ from Eq. (18). Therefore, $r\left(t_{\max }\right)<r\left(t_{\mathrm{E}}\right)$ for intermediate coupling strength (Fig. 3). Note, due to the detuning $\gamma=0.04$, the potential has a small negative mean slope, which facilitates the escape of $\varphi_{1,1}$ into the right potential well. However, during the period of time when only oscillator 2 is stimulated (between the offset of the first and the offset of the second stimulus), oscillator 1 follows oscillator 2 the quicker the larger $K$. Correspondingly, with increasing $K$ finally practically all trajectories are captured in the left potential well, so that $r\left(t_{\mathrm{E}}\right)$ gets close to 1 [Fig. 9(f)]. Furthermore, with increase in coupling strength $K$, the wells of potential $V$ get deeper and deeper, so that finally (for fixed noise amplitude) the escape rate of $\varphi_{1,1}$ tends to zero. Accordingly, also $r\left(t_{\max }\right)$ approaches 1 [Fig. 9(f)]. For values of $K$ greater than (approximately) 2, $\varphi_{1,1}$ is captured in the left potential well and cannot escape. Therefore, the CT response clustering vanishes, and $\alpha_{1}^{\max }$ tends to zero.

\section{DISCUSSION}

In this paper, I have introduced a model that makes it possible to study transient stimulus-locked phase dynamics, synchronization, and desynchronization of two coupled phase oscillators, which are stimulated at different times. Moreover, appropriate data analysis tools have been presented which enable to detect these transient dynamical processes in simulated as well as experimental data (Sec. III).

These different data analysis tools have been compared to standard data analysis techniques applied in a cross-trial manner (Sec. VII). CT cross correlation from Eq. (27) and CT standard deviation from Eq. (24) inevitably lead to severe artifacts, because they cause artificial oscillations that are not related to any real feature of the transient processes under consideration (Fig. 7). CT averaging from Eq. (21), the gold standard in evoked response studies $[6,14,15]$, may lead to massive misinterpretations since it cannot distinguish between transient response clustering and an overall decrease of the amplitude of the single responses (Fig. 6). In particular, the switching between qualitatively different responses, driven by intrinsic noise, escapes detection when the standard analysis tools are applied.

Two types of indices for analyzing stimulus-locked dynamics have been proposed and compared to each other. One sort of indices detects specific features of the CT distributions, e.g., one peak or two antiphase peaks [Eqs. (8)-(11)]: $\lambda_{j}^{(\nu)}$ and $\sigma_{n, m}^{(\nu)}$ correspond to the modulus of the $\nu$ th Fourier mode of the CT distributions from Eq. (7). In contrast, the other sort of indices is based on the Shannon entropy, and analyzes the CT distributions from Eq. (7) in a more general way by comparing them with a uniform distribution [Eqs. (12) and (13)].

It turns out, that the indices based on the Shannon entropy are not sensitive enough to detect the switching between qualitatively different responses (Sec. VI). In this respect, the Shannon entropy based indices are comparable to the CT averaged signal $\bar{x}_{j}$ from Eq. (21) and the indices based on the first Fourier mode, $\lambda_{j}^{(1)}$ and $\sigma_{n, m}^{(1)}$ : They only capture a simple reset, but not the switching dynamics, i.e., the antiphase CT response clustering and the antiphase CT branching of the trajectories of $\varphi_{1,1}$ (Fig. 5, Sec. VI). A quantity comparable to $\lambda_{j}^{(1)}$, based on a wavelet transformation and denoted as "phase-locking factor," has been used to study phase resetting in EEG signals obtained from sensory stimulation experiments $[29,22]$. Our results clearly show that it is not sufficient to exclusively compute $\lambda_{j}^{(1)}$ (Fig. 5, Sec. VI). Rather it is crucial to use higher-order indices, $\lambda_{j}^{(\nu)}$ and $\sigma_{n, m}^{(\nu)}$ with $\nu>1$ [Eqs. (8) and (9)], and related quantities [Eqs. (10) and (11)], in order to cope with complex responses.

Response decorrelation due to transient CT antiphase response clustering is robust with respect to modifications of model equation (1) and variations of its parameters.

(i) The transient dynamical phenomena studied here are not restricted to the stimuli defined by Eq. (20). In general, stimuli of first order (i.e., stimuli containing terms such as $\cos \psi_{j}$ and $\sin \psi_{j}$ ) may take the form

$$
S_{1}\left(\psi_{1}\right)=I \cos \left(\psi_{1}+\theta_{1}\right), \quad S_{2}\left(\psi_{2}\right)=I \cos \left(\psi_{2}+\theta_{2}\right)
$$

with constant phase shifts $\theta_{1}$ and $\theta_{2}$. In this case an antiphase reset is achieved and, thus, a CT response clustering occurs for

$$
t_{\mathrm{del}}=\left(\frac{\theta_{2}-\theta_{1}}{2 \pi}+0.5+k\right) T
$$

where $k$ is a small integer. For example, for $\theta_{1}=0$ and $\theta_{2}$ $=\pi$ we observe a CT response clustering for $t_{\mathrm{del}}=0, T, 2 T$, $3 T$, etc., with $T=2 \pi / \omega$. Of course, also for the stimuli given by Eq. (30) the coupling strength must not be too large with respect to the noise amplitude. Otherwise, a CT response clustering cannot occur as explained in Sec. IX. Effects of stimuli of higher order will be presented in a forthcoming study.

(ii) The CT response clustering is not limited to in-phase coupling. This can easily be shown by applying the transformation $\psi_{j} \rightarrow \psi_{j}+c_{j}$ with constant $c_{j}$. Effects caused by higher-order coupling terms, such as $\sin \left(2 \psi_{j}\right)$ or $\cos \left(2 \psi_{j}\right)$, will also be the subject of a forthcoming paper.

(iii) The CT response clustering also occurs in case of asymmetric stimulation intensities. What matters is that a stimulus is able to reset its oscillator during delivery. For this, its intensity has to be large enough compared to coupling strength and noise amplitude. Using weak stimuli, we have to be aware of the fact that in such a case the impact of stimulation typically depends on the initial phase [5]. To cope with this, we may extend the data analysis from Sec. III by performing it selectively for different ranges of the initial phases.

(iv) The duration of both stimuli does not need to be identical (cf. Fig. 1). For stimuli of different lengths the relevant parameter $t_{\text {del }}$ from Eq. (31) is the pause between their offsets, and not the pause between their onsets.

(v) An important aspect is the directionality of the couplings. In the present model, the coupling is symmetric ac- 
cording to Eq. (1). Modifying model equation (1) by including asymmetric coupling $K_{j}$ yields

$$
\dot{\psi}_{j}=\omega_{j}-K_{j} \sin \left(\psi_{j}-\psi_{k}\right)+X_{j}(t) S_{j}\left(\psi_{j}\right)+F_{j}(t),
$$

where $j, k=1,2$ and $j \neq k$. A weak asymmetry of the coupling has no dramatic impact on the CT response clustering. However, a strong asymmetry of the couplings is relevant, because, e.g., during the period of time when only oscillator 2 is stimulated (between the offset of the first stimulus and the offset of the second stimulus, see Fig. 1) oscillator 1 follows oscillator 2, the quicker the stronger oscillator 1 is coupled to oscillator 2. Asymmetries of the coupling can be revealed by asymmetries of the timing of the stimuli: Depending on whether oscillator 1 or oscillator 2 is stimulated first, the responses are qualitatively different. This effect can even be used to detect the coupling direction and will be the subject of a forthcoming communication.

Let us compare the simultaneous stimulation $\left(t_{\mathrm{del}}=0\right)$ using antiphase stimuli from Eq. (19) with the stimulation using identical stimuli from Eq. (20) with a delay of half a mean period $T\left(t_{\mathrm{del}}=0.5 T\right)$. Comparing the results from Sec. VIII with a previous study on the simultaneous stimulation [17], it turns out that in both cases a stochastic resonance occurs in a similar way. However, the two types of stimulation differ significantly concerning the effects of varying coupling strength (compare Fig. 9 with Fig. 5 in Ref. [17]). In case of the simultaneous stimulation with antiphase stimuli for vanishing coupling $(K=0)$ the amplitude of $\bar{x}_{j}$ from Eq. (21) slowly relaxes, and $\bar{x}_{j}$ runs through several cycles. Sufficiently strong coupling $K>0$ gives rise to a strong CT antiphase response clustering, which is related to strongly damped oscillatory CT averaged signals $\bar{x}_{j}$. For strong enough coupling $K$ the antiphase response clustering occurs so rapidly, such that the CT averaged signals $x_{j}$ even vanish (Fig. 5 in Ref. [17]). With increasing coupling strength the extent of CT response clustering continuously increases. In contrast, in the case of the stimulation with identical stimuli delivered with a delay of $t_{\mathrm{del}}=0.5 T$, the CT response clustering vanishes when the coupling strength gets too large (Fig. 9). Strong enough coupling prevents from an antiphase reset and, thus, from a stochastic switching between different responses (Sec. IX), so that a couplingmediated resonance occurs.

A further important difference between the simultaneous stimulation $\left(t_{\mathrm{del}}=0\right)$ and the time delayed stimulation $\left(t_{\mathrm{del}}\right.$ $=0.5 T)$ is that the former typically causes the symmetric CT response clustering [16,17], whereas the latter is typically associated with the asymmetric CT response clustering (Fig. 6). The asymmetry is due to the influence of the couplings during periods of asymmetric stimulation, i.e., stimulation of only one of the oscillators (Secs. VII A and IX).

To study transient stimulus-locked dynamics in a cluster of many coupled oscillators, we can generalize the data analysis presented in Sec. III in the following way. For illustration, let us consider a cluster of $N$ oscillators given by

$$
\dot{\psi}_{j}=\omega_{j}-\sum_{j^{\prime}=1}^{N} K_{j j^{\prime}} \sin \left(\psi_{j}-\psi_{j^{\prime}}\right)+X_{j}(t) S_{j}\left(\psi_{j}\right)+F_{j}(t),
$$

where, similar to Eq. (1), $\omega_{j}$ is the eigenfrequency of the $j$ th oscillator, $K_{j j^{\prime}}$ is the coupling constant between the $j$ th and the $j^{\prime}$ th oscillator, and $S_{j}\left(\psi_{j}\right)$ models the stimulus acting on the $j$ th oscillator. The timing of the stimuli is given by $X_{j}$. Similar to Fig. 1, we assume that the delays between the pulses affecting the different oscillators are constant across trials, respectively. The random forces $F_{j}$ are the Gaussian white noise, where $\left\langle F_{j}(t)\right\rangle=0$ and $\left\langle F_{j}(t) \quad F_{j^{\prime}}(\tilde{t})\right\rangle$ $=D \delta_{j j^{\prime}} \delta(t-\widetilde{t})$ with constant noise amplitude $D$. We can study a stimulus-locked transient dynamics in different ways.

(i) Univariate analysis with the indices from Eqs. (8) and (10): We may determine $\lambda_{j}^{(\nu)}$ from Eq. (8) and $\alpha_{j}$ from Eq. (10) for each individual oscillator $(j=1, \ldots, N)$. On the other hand, it may be advantageous to study transient dynamics on a more macroscopic, collective level of description, especially in order to estimate the impact of stimulation on synchronization processes. For this, we introduce the cluster variables

$$
Z_{k}(t)=R_{k}(t) e^{\mathrm{i} \Theta_{k}(t)}=\frac{1}{N} \sum_{j^{\prime}=1}^{N} e^{\mathrm{i} k \psi_{j^{\prime}}(t)},
$$

where $R_{k}(t)$ and $\Theta_{k}(t)$ are the corresponding real amplitude and real phase, where $0 \leqslant R_{k}(t) \leqslant 1$ for all times $t[5,18,25]$. Cluster variables are convenient for characterizing synchronized states of different type: Perfect in-phase synchronization corresponds to $R_{1}=1$, whereas an incoherent state, with uniformly distributed phases, is associated with $R_{k}=0$ ( $k$ $=1,2,3, \ldots) . R_{1}=0$ combined with large $R_{k}$ is indicative of a $k$-cluster state consisting of $k$ distinct and equally spaced clusters, where within each cluster all oscillators have similar phase. Note, in the sums in Eqs. (33) and (34) the index $j^{\prime}$ runs over all $N$ oscillators.

Similar to Eq. (8) we determine the time-dependent stimulus-locking indices of $\Theta_{k}$ by

$$
\lambda_{k}^{(\nu)}(t)=\left|\frac{1}{l} \sum_{j^{\prime}=1}^{l} \exp \left[i \nu \Theta_{k}\left(\tau_{j^{\prime}}+t\right)\right]\right| .
$$

Let us assume that before stimulation the cluster is in-phase synchronized and acts like one giant oscillator $\left(R_{1}>0\right)$. By determining $\lambda_{1}^{(1)}$, we can determine whether a stimulus resets the cluster. In this case, the stimulus causes a transient increase of $\lambda_{1}^{(1)}$ [see Fig. 4(b)]. Whether the extent of synchronization within the cluster increases or decreases during this reset can be assessed by evaluating the cross-trial average of $R_{1}$ from Eq. (34) defined by

$$
\bar{R}_{1}(t)=\frac{1}{l} \sum_{j^{\prime}=1}^{l} R_{1}\left(\tau_{j^{\prime}}+t\right)
$$

[see Eq. (21)]: A stimulus-locked increase or decrease of the synchronization of the cluster as a whole corresponds to a stimulus-locked increase or decrease of $\bar{R}_{1}$. In general, 
stimulus-locked transients of a $k$-cluster state can be studied by means of $\lambda_{k}^{(\nu)}$ and the CT average $\bar{R}_{k}$. Note, in the sums in Eqs. (35) and (36) the index $j^{\prime}$ runs over all $l$ trials.

(ii) Bivariate analysis with the indices from Eqs. (9) and (11): We may apply the data analysis method from Sec. III to all possible pairs $\left(j, j^{\prime}\right)$ of oscillators, where $j, j^{\prime}$ $=1, \ldots, N$. Especially for large $N$, it might be more appropriate to study the transient interactions on a collective level of description as described above. Furthermore, depending on the particular application we may study the interaction between different subpopulations or between the whole population and a particular oscillator with the bivariate technique from Sec. III. For example, for the study of the interaction between the whole population and a particular oscillator $j$, similar to Eq. (9), we would introduce the $n: m$ phase difference between the $j$ th oscillator and the $k$ th cluster variable according to $\left[n \psi_{j}(t)-m \Theta_{k}(t)\right] /(2 \pi) \bmod 1$ [see Eq. (5)]. For this $n: m$ phase difference we would then calculate the $n: m$ stimulus-locking indices defined by Eq. (9). In this way, it is possible to detect a stimulus-locked increase or decrease of the synchronization between the $j$ th oscillator and the $k$ th cluster variable.

The data analysis presented in Sec. III can be applied to experimental data. For discrete signals such as timing sequences of spiking neurons the phase can be estimated with linear interpolation. In an application to continuous experimental data also, the amplitudes of the oscillators have to be investigated. To this end, a relevant oscillatory signal $x_{j}(t)$, e.g., a particular brain rhythm, is extracted out of a measured signal with bandpass filtering. Instantaneous phase $\psi_{j}(t)$ and instantaneous amplitude $A_{j}(t)$ of $x_{j}(t)$ can be determined by means of the Hilbert transform $x_{j}^{H}(t)$ of $x_{j}(t)$ according to $x_{j}(t)+x_{j}^{H}(t)=A_{j}(t) \exp \left[i \psi_{j}(t)\right][27]$. The Hilbert transform is realized with a filter causing a phase shift of $\pi / 2$ for all frequencies. Alternatively, one can use the wavelet approach to determine the phase [28]. The amplitudes $A_{j}$ of the oscillators can then be averaged across trials as done in Eq. (21) with the signals. In this way, however, qualitatively different transients of the amplitudes cannot be extracted. Thus, to detect a CT clustering of amplitude transients, in the spirit of the stochastic phase resetting analysis (Sec. III), similar to Eq. (7) we can introduce CT distributions of the amplitudes with $\left\{A_{j}\left(t+\tau_{k}\right)\right\}_{k=1, \ldots, l}$ and evaluate them in a comparable way as defined by Eqs. (8) and (9) for the phases. This was not necessary here, since the amplitude of the phase oscillators is a constant. Note, when applied to experimental data, the time resolution of the stochastic data analysis methods from Sec. III is enormous, since it is only restricted by the sampling rate or, more precisely, by the time resolution of the preprocessing which yields the phases (e.g., bandpass filtering and the Hilbert transform).

Model equation (1) may be an appropriate minimal model in various fields of physics. In biophysics, neuroscience, and medicine it may apply to simple neural nets consisting of only a few neurons. In this case a single oscillator would model a single oscillatory neuron. In addition, a single oscillator may act as a simple macroscopic model for an oscilla- tory population of neurons (as discussed in Sec. VII A). Stimulation at different times is relevant for several reasons.

(i) In the nervous system, sensory information is typically transmitted via parallel pathways having different conduction times [30]. In this context, model equation (1) stands for two interconnected brain areas that generate oscillatory activity and have sensory inputs arriving at different times, comparable, e.g., to area V1 and area V5 of the visual system.

(ii) In a number of neurological diseases, for example, in multiple sclerosis conduction times may increase significantly and impair brain function.

(iii) Varying the delay $t_{\mathrm{del}}$ and administering two qualitatively different sensory stimuli or delivering electrical stimuli at different sites may serve as a key approach for studying the interactions of different brain areas during short-term information processing.

Obviously, in a next step time delays of the coupling terms have to be incorporated into the model, too.

CT averaging as defined by Eq. (21) cannot distinguish between an overall amplitude decrease of the single responses and an antiphase CT response clustering. Utilizing the typical, phenomenological reasoning applied to interpret averaged responses $[6,14]$, the exclusive consideration of the averaged signal $\bar{x}_{j}$ from Eq. (21) may give the impression that the response of a brain area is diminished (Fig. 6). This typically leads to diverse speculations concerning a reduced metabolism of this particular area or an inhibition imposed by other, hyperactive areas. However, behind such a putatively weak response, the stochastic phase resetting analysis (Sec. III) may reveal a coordinate switching between qualitatively different, strong, and long-lasting responses.

The approach presented here may identify how responses of a single brain area crucially depend on the interactions of this particular area with other areas. In particular, the stochastic phase resetting analysis may enable us to detect how brain areas may switch between different, coordinated responses to a given stimulus. Neural populations may adapt the strength of their interactions to the amount of intrinsic noise, in order to achieve an effective switching by means of stochastic resonance (Sec. VIII).

In the spinal cord the switching between coordinated responses of different neural populations is a well-known phenomenon which is essential for spinal motor control [31]. From a biological point of view, we can expect that elegant control mechanisms which turn out to be very effective in the spinal cord may also be used in parts of the nervous system that are-from the evolutionary standpoint-younger, e.g., in the neocortex. From a more pragmatic point of view we may suspect that the assumption behind the CT averaging, Eq. (22), acts as a self-fulfilling prophecy: Looking for the only stereotyped response in an inventory of responses without using a self-consistency criterion produces artifacts, inevitably. The data analysis methods from Sec. III are already being applied to MEG/EEG signals and the cerebral current source density.

\section{ACKNOWLEDGMENTS}

This study was supported by the Volkswagen Foundation (Grant No. 76761) and the German Israeli Foundation (Grant No. I-667-81.1/2000). 
[1] H. Haken, Advanced Synergetics (Springer, Berlin, 1983); A. S. Pikovsky et al., Synchronization. A Universal Concept in Nonlinear Sciences (Cambridge University Press, Cambridge, 2001).

[2] Y. Kuramoto, Chemical Oscillations, Waves, and Turbulence (Springer, Berlin, 1984).

[3] A. T. Winfree, The Geometry of Biological Time (Springer, Berlin, 1980).

[4] H. Steriade et al., Thalamic Oscillations and Signaling (Wiley, New York, 1990).

[5] P. A. Tass, Phase Resetting in Medicine and Biology. Stochastic Modelling and Data Analysis (Springer, Berlin, 1999).

[6] K. H. Chiappa, Evoked Potentials in Clinical Medicine (Raven Press, New York, 1983).

[7] M.G. Rosenblum et al., Phys. Rev. Lett. 76, 1804 (1996).

[8] K. Josić and D.J. Mar, Phys. Rev. E 64, 056234 (2001); M.A. Zaks et al., ibid. 65, 026212 (2002).

[9] I.Z. Kiss and J.L. Hudson, Phys. Rev. E 64, 046215 (2001); I.Z. Kiss et al., Phys. Rev. Lett. 88, 238301 (2002).

[10] P. Tass et al., Phys. Rev. Lett. 81, 3291 (1998); P.A. Tass et al., ibid. 90, 088101 (2003).

[11] A.B. Neiman et al., Phys. Rev. Lett. 83, 4896 (1999).

[12] A.B. Neiman et al., Phys. Rev. E 58, 7118 (1998); J.A. Freund et al., Europhys. Lett. 50, 8 (2000); A.B. Neiman and D.F. Russell, Phys. Rev. Lett. 88, 138103 (2002); S. Bahar et al., Phys. Rev. E 65, 050901 (2002).

[13] R. L. Stratonovich, Topics in the Theory of Random Noise
(Gordon and Breach, New York, 1963).

[14] G.D. Dawson, Electroencephalogr Clin. Neurophysiol. Suppl. 44, 153 (1954).

[15] M. Hämäläinen et al., Rev. Mod. Phys. 65, 413 (1993).

[16] P.A. Tass, Europhys. Lett. 59, 199 (2002).

[17] P. A. Tass, Chaos 13, 364 (2003).

[18] H. Daido, Prog. Theor. Phys. 88, 1213 (1992).

[19] R. K. Otnes and L. Enochson, Digital Time Series Analysis (Wiley, New York, 1972).

[20] R. Hari and R. Salmelin, TINS 20, 44 (1997).

[21] B. Sayers et al., Nature (London) 247, 481 (1974).

[22] S. Makeig et al., Science 295, 690 (2002).

[23] Handbook of Biological Physics, edited by C. Gielen and F. Moss (Elsevier, Amsterdam, 2001), Vol. IV.

[24] C. W. Gardiner, Handbook for Stochastic Methods for Physics, Chemistry and the Natural Sciences, 2nd ed. (Springer, Berlin, 1985).

[25] P. Tass, Phys. Rev. E 56, 2043 (1997).

[26] L. Gammaitoni et al., Rev. Mod. Phys. 70, 223 (1998).

[27] P. Panter, Modulation, Noise, and Spectral Analysis (McGrawHill, New York, 1965).

[28] R. Quian Quiroga et al., Phys. Rev. E 65, 041903 (2002).

[29] C. Tallon-Baudry et al., J. Neurosci. 16, 4240 (1996).

[30] Principles of Neural Science, edited by E. R. Kandel et al. (Appleton \& Lange, Norwalk, CT, 2000) Chap. 30, pp. 440466.

[31] H. Forssberg et al., Brain Res. 85, 103 (1975). 